\title{
Adaptive Backstepping Finite Time Attitude Control of Reentry RLV with Input Constraint
}

\author{
Fang Wang, Qun Zong, and Bailing Tian \\ College of Electric Engineering and Automation, Tianjin University, No. 92, Weijin Road, Nankai District, Tianjin 300072, China \\ Correspondence should be addressed to Fang Wang; wangfang@ysu.edu.cn
}

Received 2 August 2013; Revised 16 December 2013; Accepted 17 December 2013; Published 29 January 2014

Academic Editor: Rongni Yang

Copyright (C) 2014 Fang Wang et al. This is an open access article distributed under the Creative Commons Attribution License, which permits unrestricted use, distribution, and reproduction in any medium, provided the original work is properly cited.

\begin{abstract}
This paper presents the finite time attitude tracking control problem of reusable launch vehicle (RLV) in reentry phase under input constraint, model uncertainty, and external disturbance. A control-oriented model of rotational dynamics is developed and used for controller design for the complex coupling of the translational and rotational dynamics. Firstly, fast terminal sliding mode control is incorporated into backstepping control to design controller considering model uncertainty and external disturbance. The "explosion of terms" problem inherent in backstepping control is eliminated by a robust second order filter. Secondly, the control problem in the presence of input constraint is further considered, and a constrained adaptive backstepping fast terminal sliding mode control scheme is developed. At the control design level, adaptive law is employed to estimate the unknown norm bound of lumped uncertainty with the reduction of computational burden. The Lyapunov-based stability analysis of the closed-loop system is carried out. The control performance is presented via the simulation of six-degree-of-freedom (6-DOF) model of RLV.
\end{abstract}

\section{Introduction}

Reusable launch vehicle (RLV) is designed to dramatically reduce the cost of accessing space by recovering and reusing after each mission. A major challenge posed in such mission is atmospheric reentry. In the reentry phase, a number of stringent constraints come into action in which the constraints include constraints on heat flux structural load, and they restrict the vehicle to fly within a narrow flight corridor [1]. Advanced guidance and control technologies are critical for meeting safety, reliability, and cost requirements for RLV. These methods have received considerable attention during the last decades. The main task of entry guidance is the on-board correction of the flight path to compensate for disturbance and model uncertainties. The effective guidance law has been designed to compute the commanded angle of attack and bank angle. Then, the emphasis is to design an appropriate attitude control system to track the guidance command, which is the focus of this paper. Several approaches have been proposed in the past, such as gain scheduling (GS) $[2,3]$, dynamic inversion (DI) technique [4-6], trajectory linearization control (TLC) [7-9], sliding mode control (SMC) $[10,11]$, and state-dependent Riccati equation (SDRE) control $[12,13]$. They provided good control performance, but input constraint was not taken into account in the above research.

Input saturation often severely limits system performance, giving undesirable inaccuracy or leading to instability $[14,15]$, and it is a potential problem for the actuators of control systems. Input saturation is often encountered in practical applications due to the fact that it is usually impossible to implement unlimited control signals [16]. Physical input saturation on hardware dictates that the magnitude of control signal is always constrained [17]. In flight control system, aircraft body may change seriously when input saturation occurs, and it may lead the aircraft to disintegrate. In order to avoid the above problem for the flight control sytem, it is necessary to study the problem of controller design subject to input constraint. It raises challenges for control system design with the nonanalytic actuator nonlinear dynamics and unknown exact actuator nonlinear functions. In addition, the consideration of model uncertainty and external disturbance makes it more difficult.

Though a lot of methods are proposed to handle input constraint in theory [18-21], only limited studies are concerned about attitude control problem with control limits due 
to the complicated nonlinear dynamics of hypersonic vehicle systems. Antiwindup control (AWC) was developed to handle input constraint $[22,23]$, but the stability of closed-loop system is difficult to be analyzed theoretically [24]. Model predictive control (MPC) has been used popularly because of its inherent capability of tackling input constraint directly during controller design procedure [25-27]. However, it depends on the real-time receding horizon optimization. The main barrier of its application to hypersonic vehicle is online optimization and the determination of time-domain step size [28]. Furthermore, there are other methods to handle input constraint for flight control system. A direct model reference adaptive control was proposed for F-16 aircraft in the presence of position limited, unknown parameters and matched uncertainties [29]. Direct adaptive control and theta-D control were presented to spacecraft with two classes of input saturations [30]. Those two methods were robust enough to handle nominal operating conditions and input saturation situations. An adaptive backstepping controller was designed for the F-16 [31], where command filters were used to implement constraints on control surfaces and virtual control states, whereas it only considered linear parameterization uncertainty. An integral backstepping control with positive control parameters was developed to reduce the guaranteed maximum torque upper bound for spacecraft [32]. An adaptive backstepping approach was applied to the flight control of longitudinal aircraft dynamics that directly accommodated magnitude, rate, and bandwidth limits on aircraft states and actuators [33]. It is noted that the external disturbance was not considered.

Besides, input constraint, uncertain inertia matrix, and external disturbance may also reduce the accuracy of the control system, and they should not be ignored during the control system design. It is not hard to only handle one of the above problems with some control approach, but once all the above factors are considered, the situation will be hard to deal with. Therefore, it is necessary to focus on the attitude tracking control problem with the simultaneous consideration of input constraint, uncertain inertia matrix, and external disturbance. Moreover, since the attitude dynamics of RLV are highly nonlinear, the problem of how to design a high-precision finite time attitude control law is a challenging work. Many nonlinear control approaches have been employed to solve the finite time attitude tracking control problem. SMC is one of the most powerful techniques to handle nonlinear systems with uncertainties and bounded external disturbances. It has been adopted in many previous works for attitude control design of hypersonic vehicle [10,11]. Backstepping control was proposed by Kristic et al. [34]. It is a typical nonlinear control approach and a powerful tool to deal with unmatched uncertainty [35]. Some researches focus on the combination of SMC and backstepping control [3641]. The conventional linear sliding mode surface is employed for the general SMC. The tracking error converges to the equilibrium points asymptotically after the system reaches the sliding mode manifolds; this convergence process will cost infinite time theoretically. For the hypersonic vehicle, slow convergence may affect the control precision to some extent. Nonlinear function is introduced at the sliding mode surface design level and terminal sliding mode (TSM) control scheme is proposed to enhance the convergence velocity. TSM control can guarantee the convergence of tracking error in finite time, and it has better control precision than the linear sliding mode control.

In this research, we focus on the attitude control problem that a RLV in reentry phase will be driven to track the guidance command where input constraint, model uncertainty, and external disturbance are taken into account. The control-oriented model is firstly established. Since it is a strict-feedback system with unmatched uncertainty, an adaptive backstepping fast terminal sliding mode controller is developed to handle model uncertainty and external disturbance. Furthermore, a constrained adaptive backstepping fast terminal sliding mode control scheme is proposed to tackle input constraint, model uncertainty, and external disturbance. Then, the stability of the closed-loop system is proved through Lyapunov technique. The 6-DOF simulations are performed to evaluate the effectiveness of the proposed control strategy.

The outline of this paper is as follows. In Section 2, the 6-DOF equations of motion of RLV and the control-oriented model are stated. Then, adaptive backstepping fast terminal sliding mode control strategy is developed with inertia matrix uncertainty and external disturbance in Section 3. To implement the constrained input, the constrained adaptive backstepping fast terminal sliding mode control strategy is designed in Section 4. Next, simulations applying two control schemes to the RLV in reentry phase are done in Section 5. At last, conclusions and future work are given in Section 6.

\section{Reusable Launch Vehicle Model}

In this section, the 6-DOF equations of motion of RLV during reentry phase are described, and the control-oriented model is derived for control design.

2.1. Six-Degree-Of-Freedom Equations of Motion. The motion of 6-DOF unpowered rigid flight vehicle can be separated into center-of-mass motion (translational motion) which is referenced to a flight-path coordinate system, and the motion that is around center-of-mass (rotational or attitude motion), is referenced to a vehicle-body-fixed coordinate system.

The center-of-mass motion is caused by the forces that act on the vehicle. It is used for generating trajectory and designing guidance law. Most applications assume steady coordinated turns such that the sideslip angle is zero. The equations of translational motion are given by [42]

$$
\begin{gathered}
\dot{h}=v \sin \gamma, \\
\dot{\phi}=\frac{v \cos \gamma \sin \chi}{\left(R_{E}+h\right) \cos \theta}, \\
\dot{\theta}=\frac{v}{\left(R_{E}+h\right)} \cos \gamma \cos \chi, \\
\dot{v}=-\frac{D}{m}-g \sin \gamma+\Omega_{E}^{2}\left(R_{E}+h\right) \cos \theta \\
\cdot(\sin \gamma \cos \theta-\cos \gamma \sin \theta \cos \chi),
\end{gathered}
$$




$$
\begin{gathered}
\dot{\gamma}=\frac{L \cos \sigma}{m v}-\left(\frac{g}{v}-\frac{v}{\left(R_{E}+h\right)}\right) \cos \gamma+2 \Omega_{E} \cos \theta \sin \chi \\
+\frac{\Omega_{E}^{2}\left(R_{E}+h\right)}{v} \cos \theta \cdot(\cos \gamma \cos \theta+\sin \gamma \sin \theta \cos \chi), \\
\dot{\chi}=\frac{L \sin \sigma}{m v \cos \gamma}+\frac{v}{\left(R_{E}+h\right)} \cos \gamma \sin \chi \tan \theta \\
-2 \Omega_{E}(\tan \gamma \cos \theta \cos \chi-\sin \theta) \\
+\frac{\Omega_{E}^{2}\left(R_{E}+h\right)}{v \cos \gamma} \sin \theta \cos \theta \sin \chi
\end{gathered}
$$

where $h, \phi, \theta, v, \gamma$, and $\chi$ are altitude, latitude, longitude, velocity, flight path angle, and heading angle, respectively. $L$ and $D$ are lift and drag, respectively. $g$ is the gravity acceleration $\left(g=\mu /\left(R_{E}+h\right)^{2}\right.$ with $\mu$ being the Earth gravity constant). $\Omega_{E}$ is the Earth angular speed, and $R_{E}$ is radius of the Earth.

The rotational equations governing the rigid-body attitude dynamic of the vehicle during the reentry flight are given as follows [26]:

$$
\begin{aligned}
& \dot{\alpha}=-p \cos (\alpha) \tan (\beta)+q-r \sin (\alpha) \tan (\beta) \\
& -\frac{\cos (\sigma)}{\cos (\beta)}\left[\dot{\gamma}-\dot{\phi} \cos (\chi)-\left(\dot{\theta}+\Omega_{E}\right) \cos (\phi) \sin (\chi)\right] \\
& +\frac{\sin (\sigma)}{\cos (\beta)}[\dot{\chi} \cos (\gamma)-\dot{\phi} \sin (\chi) \sin (\gamma)] \\
& +\frac{\sin (\sigma)}{\cos (\beta)}\left[\phi+\left(\dot{\theta}+\Omega_{E}\right)(\cos (\phi) \cos (\chi) \sin (\gamma))\right. \\
& -\sin (\phi) \cos (\gamma)] \\
& \dot{\beta}=p \sin (\alpha)-r \cos (\alpha) \\
& +\sin (\sigma)\left[\dot{\gamma}-\dot{\phi} \cos (\chi)+\left(\dot{\theta}+\Omega_{E}\right) \cos (\phi) \sin (\chi)\right] \\
& +\cos (\sigma)[\dot{\chi} \cos (\gamma)-\dot{\phi} \sin (\chi) \sin (\gamma)] \\
& -\cos (\sigma)\left(\dot{\theta}+\Omega_{E}\right) \\
& \cdot(\cos (\phi) \cos (\chi) \sin (\gamma)-\sin (\phi) \cos (\gamma)) \text {, } \\
& \dot{\sigma}=-p \cos (\alpha) \cos (\beta)-q \sin (\beta)-r \sin (\alpha) \cos (\beta) \\
& +\dot{\alpha} \sin (\beta)-\dot{\chi} \sin (\gamma)-\dot{\phi} \sin (\chi) \cos (\gamma) \\
& +\left(\dot{\theta}+\Omega_{E}\right)[\cos (\phi) \cos (\chi) \cos (\gamma)+\sin (\phi) \sin (\gamma)], \\
& \dot{p}=\frac{I_{z z} M_{x}}{I_{x x} I_{z z}-I_{x z}^{2}}+\frac{I_{x z} M_{z}}{I_{x x} I_{z z}-I_{x z}^{2}} \\
& +\frac{\left(I_{x x}-I_{y y}+I_{z z}\right) I_{x z}}{I_{x x} I_{z z}-I_{x z}^{2}} p q+\frac{\left(I_{y y}-I_{z z}\right) I_{z z}-I_{x z}^{2}}{I_{x x} I_{z z}-I_{x z}^{2}} q r
\end{aligned}
$$

$$
\begin{gathered}
\dot{q}=\frac{M_{y}}{I_{y y}}+\frac{I_{x z}}{I_{y y}}\left(r^{2}-p^{2}\right)+\frac{I_{z z}-I_{x x}}{I_{y y}} p r, \\
\dot{r}=\frac{I_{x z} M_{x}}{I_{x x} I_{z z}-I_{x z}^{2}}+\frac{I_{x x} M_{z}}{I_{x x} I_{z z}-I_{x z}^{2}} \\
+\frac{\left(I_{x x}-I_{y y}\right) I_{x x}+I_{x z}^{2}}{I_{x x} I_{z z}-I_{x z}^{2}} p q+\frac{\left(I_{y y}-I_{x x}-I_{z z}\right) I_{x z}}{I_{x x} I_{z z}-I_{x z}^{2}} q r,
\end{gathered}
$$

where $\alpha, \beta$, and $\sigma$ denote, respectively, angle of attack (AOA), sideslip angle, and bank angle. $p, q$, and $r$ are roll rate, pitch rate and yaw rate, respectively. $M_{x}, M_{y}$, and $M_{z}$ are rolling moment, pitching moment, and yawing moment, respectively. $I_{i_{0} j_{0}}\left(i_{0}=x, y, z ; j_{0}=x, y, z\right)$ are inertia. The expressions of the lift and drag are as follow: $L=\bar{q} S C_{L}(\alpha)$, $D=\bar{q} S C_{D}(\alpha)$, where $C_{L}(\alpha)$ and $C_{D}(\alpha)$ are the lift coefficient and drag coefficient, respectively, and they are the functions of AOA. Aerodynamic reference area of vehicle is given by $S=2690 \mathrm{ft}^{2}$, and dynamic pressure is given by $\bar{q}=$ $0.5 \rho v^{2}$. The specific aerodynamic data are referred to [43], and the atmospheric model is referred to in 1976 US Standard Atmosphere.

2.2. Control-Oriented Model. It is noted that (2) are nonlinear, and the states are coupled with trajectory states, so a controloriented model is developed for controller design succinctly. In fact, the rotational motions of RLV are much faster than translational motions and the motion of the Earth. Time derivatives of both position and direction of the velocity and the Earth's angular velocity are considered to be negligible with respect to the rotational motion, resulting in the following:

$$
\begin{gathered}
\dot{\alpha}=-p \cos \alpha \tan \beta+q-r \sin \alpha \tan \beta+\Delta f_{11}, \\
\dot{\beta}=p \sin \alpha-r \cos \alpha+\Delta f_{12}, \\
\dot{\sigma}=-p \cos \alpha \cos \beta-q \sin \beta-r \sin \alpha \cos \beta+\Delta f_{13},
\end{gathered}
$$

where $\Delta f_{1 i}(i=1,2,3)$ denote uncertainties induced by model simplification.

The equations (2) can be written as

$$
(\mathbf{I}+\Delta \mathbf{I}) \dot{\boldsymbol{\omega}}=-\mathbf{\Omega}(\mathbf{I}+\Delta \mathbf{I}) \boldsymbol{\omega}+\mathbf{u}+\Delta \mathbf{D}_{1},
$$

where $\Delta \mathbf{I}$ and $\Delta \mathbf{D}_{1}=\left[\begin{array}{lll}d_{21} & d_{22} & d_{23}\end{array}\right]^{T}$ denote inertia matrix uncertainty and external disturbance, respectively.

Equations (3)-(4) are used for controller design, and they can be expressed as

$$
\begin{gathered}
\dot{\mathbf{\Omega}}=\mathrm{R} \boldsymbol{\omega}+\Delta \mathbf{f}_{1}, \\
\dot{\boldsymbol{\omega}}=-\mathrm{I}^{-1} \Phi \mathrm{I} \boldsymbol{\omega}+\mathrm{I}^{-1} \mathbf{u}+\Delta \mathrm{D}_{2} .
\end{gathered}
$$

Here $\Delta \mathbf{D}_{2}=\mathbf{I}^{-1}\left(\Delta \mathbf{D}_{1}-\Delta \mathbf{I} \dot{\boldsymbol{\omega}}-\boldsymbol{\Phi} \Delta \mathbf{I} \boldsymbol{\omega}\right)$ and $\boldsymbol{\omega}=\left[\begin{array}{lll}p & q & r\end{array}\right]^{T}$ is attitude angular rate vector. $\mathbf{\Omega}=\left[\begin{array}{lll}\alpha & \beta & \sigma\end{array}\right]^{T}$ is attitude angle vector. $\Delta \mathbf{f}_{1}=\left[\begin{array}{lll}\Delta f_{11} & \Delta f_{12} & \Delta f_{13}\end{array}\right]^{T}$ is the uncertain vector 
induced by model simplification. $\mathbf{u}=\left[\begin{array}{lll}M_{x} & M_{y} & M_{z}\end{array}\right]^{T}$ is the control input vector. The matrixes $\mathbf{I}, \boldsymbol{\Phi}$, and $\mathbf{R}$ are as follows

$$
\begin{gathered}
\mathbf{I}=\left[\begin{array}{ccc}
I_{x x} & -I_{x y} & -I_{x z} \\
-I_{x y} & I_{y y} & -I_{y z} \\
-I_{x z} & -I_{y z} & I_{z z}
\end{array}\right], \\
\boldsymbol{\Phi}=\left[\begin{array}{ccc}
0 & -r & q \\
r & 0 & -p \\
-q & p & 0
\end{array}\right], \\
\mathbf{R}=\left[\begin{array}{ccc}
-\cos \alpha \tan \beta & 1 & -\sin \alpha \tan \beta \\
\sin \alpha & 0 & -\cos \alpha \\
-\cos \alpha \cos \beta & -\sin \beta & -\sin \alpha \cos \beta
\end{array}\right] .
\end{gathered}
$$

The dynamics (5)-(6) possess a strict-feedback formulation, which makes the backstepping technique applicable. For the fast and finite-time convergence, and high steady-state tracking precision, the fast terminal sliding mode control is integrated with backstepping control to design the control scheme.

\section{Adaptive Backstepping Fast Terminal Sliding Mode Control (ABFTSMC) Design}

In this section, for the control-oriented model (COM) (5)(6), attitude tracking controller is developed taking model uncertainty and external disturbance into account.

Assumption 1. Every term of unknown external disturbance $\Delta \mathbf{D}_{1}$ is bounded by $\left|d_{1 i}\right| \leq \eta_{1 i}$, and $\Delta \mathbf{D}_{1}$ satisfies the following inequality:

$$
\left\|\mathbf{\Delta}_{1}\right\| \leq \bar{d}_{1}=\sqrt{\sum_{i=1}^{3} \eta_{1 i}^{2}}
$$

where $\eta_{1 i}(i=1,2,3)$ are known positive constants.

Assumption 2. The unknown term $\Delta \mathbf{f}_{1}$ is bounded and satisfies the following inequality:

$$
\left\|\Delta \mathbf{f}_{1}\right\| \leq \rho_{10}
$$

where $\rho_{10}$ is an unknown positive constant.

The control objective is to design controller for the COM (5)-(6) to guarantee that the output $\Omega$ tracks the guidance command $\boldsymbol{\Omega}_{d}$ in spite of model uncertainty and external disturbance, with assumption that every element of $\boldsymbol{\Omega}_{d}$ and its first order time derivative are continuous and bounded.

Following the backstepping design procedure, the COM (5)-(6) is decomposed into attitude angle subsystem and attitude angular rate subsystem. The control scheme design begins from attitude angle subsystem (5) where the virtual control input is developed and used as the reference command for the angular rate subsystem (6). Then, the actual control input is designed. The concrete design procedure is described in the following two subsections.
3.1. Controller Design for Attitude Angle Subsystem. Attitude angular rate is chosen as the virtual control input. Tracking errors of attitude angle and attitude angular rate are defined as follows:

$$
\begin{aligned}
& \mathbf{z}_{1}=\boldsymbol{\Omega}-\boldsymbol{\Omega}_{d}, \\
& \mathbf{z}_{2}=\boldsymbol{\omega}-\boldsymbol{\omega}_{d},
\end{aligned}
$$

where $\boldsymbol{\Omega}_{d}=\left[\begin{array}{lll}\alpha_{d} & \beta_{d} & \sigma_{d}\end{array}\right]^{T}$ is the guidance command that is generated by the guidance system. $\omega_{d}$ is the reference command of attitude angular rate subsystem, and it is the virtual control input to be designed.

Inspired by the work in [44], a second order sliding mode manifold for attitude angle subsystem is chosen as

$$
\mathbf{s}_{1}=\dot{\mathbf{z}}_{1}+\lambda_{1} \mathbf{z}_{1}+\lambda_{2} \mathbf{z}_{1}^{m_{1} / n_{1}}
$$

where $\lambda_{1}, \lambda_{2}>0, m_{1}, n_{1}\left(m_{1}<n_{1}\right)$ are positive odd integers.

The virtual control input $\omega_{d}$ is designed as the following formation

$$
\boldsymbol{\omega}_{d}=\boldsymbol{\omega}_{d \mathrm{eq}}+\boldsymbol{\omega}_{d s}
$$

where $\boldsymbol{\omega}_{d \mathrm{eq}}$ and $\boldsymbol{\omega}_{d s}$ are equivalent control and nonlinear control, respectively.

Based on (5) and (10)-(13), we have

$$
\begin{aligned}
\mathbf{s}_{1}= & \mathbf{R}\left(\boldsymbol{\omega}_{d \mathrm{eq}}+\boldsymbol{\omega}_{d s}\right)+\mathbf{R} \mathbf{z}_{2}+\Delta \mathbf{f}_{1}-\dot{\boldsymbol{\Omega}}_{d} \\
& +\lambda_{1} \mathbf{z}_{1}+\lambda_{2} \mathbf{z}_{1}^{m_{1} / n_{1}}
\end{aligned}
$$

The uncertain term $\Delta \mathbf{f}_{1}$ and $\mathbf{z}_{2}$ are not regarded, besides, let $\mathbf{s}_{1}=\mathbf{0}$; then $\boldsymbol{\omega}_{\text {deq }}$ can be designed as

$$
\boldsymbol{\omega}_{d \mathrm{eq}}=\mathbf{R}^{-1}\left(\dot{\boldsymbol{\Omega}}_{d}-\lambda_{1} \mathbf{z}_{1}-\lambda_{2} \mathbf{z}_{1}^{m_{1} / n_{1}}\right) .
$$

Substituting (15) into (14), if the controller can carry out the high-precision tracking in the next step, that is, $\mathbf{z}_{2}=\dot{\mathbf{z}}_{2}=\mathbf{0}$, then we have

$$
\mathbf{s}_{1}=\mathbf{R} \boldsymbol{\omega}_{d s}+\Delta \mathbf{f}_{1}
$$

Using the derivative of $\mathbf{s}_{1}$ with respect to time, then

$$
\dot{\mathbf{s}}_{1}=\mathbf{R} \dot{\boldsymbol{\omega}}_{d s}+\dot{\mathbf{R}} \boldsymbol{\omega}_{d s}+\Delta \dot{\mathbf{f}}_{1}
$$

Based on Assumption 2, the following assumption holds.

Assumption 3. The term $\dot{\mathbf{R}} \boldsymbol{\omega}_{d s}+\Delta \dot{\mathbf{f}}_{1}$ satisfies $\left\|\dot{\mathbf{R}} \boldsymbol{\omega}_{d s}+\Delta \dot{\mathbf{f}}_{1}\right\| \leq \rho_{1}$, where $\rho_{1}$ is an unknown positive constant.

Since $\rho_{1}$ needs to be estimated online, the representation $\hat{\rho}_{1}$ will be applied to denote the estimation, and an adaptive law is used to update $\hat{\rho}_{1}$.

From (17), the nonlinear control term $\omega_{d s}$ can be designed as

$$
\boldsymbol{\omega}_{d s}=-\left(\hat{\rho}_{1}+\varepsilon_{1}\right) \mathbf{R}^{-1} \int \operatorname{sgn}\left(\mathbf{s}_{1}\right) d t
$$


and the adaptive law for $\widehat{\rho}_{1}$ is

$$
\dot{\hat{\rho}}_{1}=\mu_{1}\left\|\mathbf{s}_{1}\right\|
$$

where $\varepsilon_{1}, \mu_{1}>0$ are parameters to be designed.

The Lyapunov candidate function is chosen as

$$
V_{1}=0.5 \mathbf{s}_{1}^{T} \mathbf{s}_{1}+\frac{1}{2 \mu_{1}} \widetilde{\rho}_{1}^{2},
$$

where $\widetilde{\rho}_{1}$ is the estimation error of $\rho_{1}, \widetilde{\rho}_{1}=\widehat{\rho}_{1}-\rho_{1}$.

From (17)-(20), the time derivative of $V_{1}$ satisfies

$$
\begin{aligned}
\dot{V}_{1}= & \mathbf{s}_{1}^{T} \dot{\mathbf{s}}_{1}+\frac{1}{\mu_{1}} \widetilde{\rho}_{1} \dot{\hat{\rho}}_{1} \\
= & \mathbf{s}_{1}^{T}\left(-\left(\widehat{\rho}_{1}+\varepsilon_{1}\right) \mathbf{R}^{-1} \operatorname{sgn}\left(\mathbf{s}_{1}\right)+\dot{\mathbf{R}} \boldsymbol{\omega}_{d s}+\Delta \dot{\mathbf{f}}_{1}\right) \\
& +\widehat{\rho}_{1}\left\|\mathbf{s}_{1}\right\|-\rho_{1}\left\|\mathbf{s}_{1}\right\| \\
\leq & -\varepsilon_{1}\left\|\mathbf{s}_{1}\right\| .
\end{aligned}
$$

Based on the above inequality, if $\left\|\mathbf{s}_{1}\right\| \neq 0$, then $\dot{V}_{1}<0$, and $\mathbf{s}_{2}$ is asymptotically stable.

By integrating (19), $\widehat{\rho}_{1}(t)=\widehat{\rho}_{1}(0)+\mu_{1} \int_{0}^{t}\left\|\mathbf{s}_{1}\right\| d t$. When $\left\|\mathbf{s}_{1}\right\| \neq 0$ and if we choose the appropriate values of $\hat{\rho}_{1}(0)$ and $\mu_{1}$ there is time $t_{1}$, for any random time $t>t_{1}, \hat{\rho}_{1}(t) \geq \rho(t)$ holds. So, $\tilde{\rho}(t) \geq 0\left(t>t_{1}\right)$ [45]. From (19) and (21), $\mathbf{s}_{1}^{T} \dot{\mathbf{s}}_{1}+$ $\tilde{\rho}_{1}\left\|\mathbf{s}_{1}\right\| \leq-\varepsilon_{1}\left\|\mathbf{s}_{1}\right\| ;$ moreover, $\left\|\mathbf{s}_{1}^{T} \dot{\mathbf{s}}_{1}\right\| \geq \varepsilon_{1}\left\|\mathbf{s}_{1}\right\|+\widetilde{\rho}_{1}\left\|\mathbf{s}_{1}\right\|$; thus,

$$
\left\|\dot{\mathbf{s}}_{1}\right\| \geq \varepsilon_{1}+\tilde{\rho}_{1}>0 .
$$

It indicates that $\mathbf{s}_{1}$ will converge to origin in finite time. After it reaches the surface $\mathbf{s}_{1}=0, \mathbf{z}_{1}$ and $\dot{\mathbf{z}}_{1}$ will reach the origin in finite time [44].

3.2. Controller Design for Attitude Angular Rate Subsystem. Motivated by the work in [44], a second order sliding mode manifold for attitude angular rate subsystem is chosen as

$$
\mathbf{s}_{2}=\dot{\mathbf{z}}_{2}+\lambda_{3} \mathbf{z}_{2}+\lambda_{4} \mathbf{z}_{2}^{m_{2} / n_{2}}
$$

where $\lambda_{3}, \lambda_{4}>0, m_{2}, n_{2}\left(m_{2}<n_{2}\right)$ are positive odd integers.

The actual control input $\mathbf{u}$ is designed as the following formation:

$$
\mathbf{u}=\mathbf{u}_{\mathrm{eq}}+\mathbf{u}_{s}
$$

where $\mathbf{u}_{\mathrm{eq}}$ and $\mathbf{u}_{s}$ are equivalent control and nonlinear control, respectively.

Based on (6), (11), and (24), (23) becomes

$$
\begin{aligned}
\mathbf{s}_{2}= & -\mathbf{I}^{-1} \boldsymbol{\Phi} \mathbf{I} \boldsymbol{\omega}+\mathbf{I}^{-1}\left(\mathbf{u}_{\mathrm{eq}}+\mathbf{u}_{s}\right)+\Delta \mathbf{D}_{2}-\dot{\boldsymbol{\omega}}_{d} \\
& +\lambda_{3} \mathbf{z}_{2}+\lambda_{4} \mathbf{z}_{2}^{m_{2} / n_{2}} .
\end{aligned}
$$

Remark 4. The time derivative of $\boldsymbol{\omega}_{d}$ needs to be computed from equation (25). Due to the nonlinearity and uncertainty of the COM (5)-(6), it is difficult to obtain the time derivative of virtual control input $\omega_{d}$ even if it can be analytically computed. For "explosion of terms" problem results from the repeated differentiations of desired virtual control in backstepping control, dynamic surface control was presented through introducing a first order filtering of the synthetic input at each step to estimate the time derivative of virtual control input [46, 47]. Moreover, command filters were proposed in $[48,49]$ to avoid analytical calculation of the time derivatives of virtual control inputs in the backstepping procedure.

In this study, a second order filter is employed to avoid the analytic computation to eliminate the "explosion of terms" problem. The second order filter is depicted by [50]

$$
\begin{gathered}
\dot{\overline{\boldsymbol{\omega}}}_{d}=-\frac{\widehat{\boldsymbol{\omega}}_{d}-\boldsymbol{\omega}_{d}}{\tau_{1}}-\frac{\psi_{1}\left(\hat{\boldsymbol{\omega}}_{d}-\boldsymbol{\omega}_{d}\right)}{\left\|\hat{\boldsymbol{\omega}}_{d}-\boldsymbol{\omega}_{d}\right\|+\sigma_{1}}, \\
\dot{\overline{\boldsymbol{\omega}}}_{d}=-\frac{\overline{\boldsymbol{\omega}}_{d}-\dot{\widehat{\boldsymbol{\omega}}}_{d}}{\tau_{2}}-\frac{\psi_{2}\left(\overline{\boldsymbol{\omega}}_{d}-\dot{\widehat{\boldsymbol{\omega}}}_{d}\right)}{\left\|\overline{\boldsymbol{\omega}}_{d}-\dot{\overline{\boldsymbol{\omega}}}_{d}\right\|+\sigma_{2}},
\end{gathered}
$$

where $\tau_{j}$ is the filter time constant while $\psi_{j}$ and $\sigma_{j}$ are positive constants $(j=1,2)$. The filter estimation errors are defined as $\mathbf{e}_{f 1}=\widehat{\boldsymbol{\omega}}_{d}-\boldsymbol{\omega}_{d}, \mathbf{e}_{f 2}=\overline{\boldsymbol{\omega}}_{d}-\dot{\overline{\boldsymbol{\omega}}}_{d}$.

If the uncertain term $\Delta \mathbf{D}_{2}$ is not considered, let $\mathbf{s}_{2}=\mathbf{0}$; that is,

$$
\mathbf{s}_{2}=-\mathbf{I}^{-1} \boldsymbol{\Phi} \mathbf{\omega}+\mathbf{I}^{-1}\left(\mathbf{u}_{\mathrm{eq}}+\mathbf{u}_{s}\right)-\dot{\boldsymbol{\omega}}_{d}+\lambda_{3} \mathbf{z}_{2}+\lambda_{4} \mathbf{z}_{2}^{m_{2} / n_{2}}
$$

Then the equivalent control $\mathbf{u}_{\text {deq }}$ can be designed as

$$
\mathbf{u}_{\mathrm{eq}}=\mathbf{I}\left(\mathbf{I}^{-1} \boldsymbol{\Phi} \mathbf{I} \boldsymbol{\omega}+\overline{\boldsymbol{\omega}}_{d}-\lambda_{3} \mathbf{z}_{2}-\lambda_{4} \mathbf{z}_{2}^{m_{2} / n_{2}}\right)
$$

Considering the uncertainty $\Delta \mathbf{D}_{2}$, and substituting (28) into (27), $\mathbf{s}_{2}=\mathbf{I}^{-1} \mathbf{u}_{s}+\Delta \mathbf{D}_{2}$. Using the derivative of $\mathbf{s}_{2}$, with respect to time, then

$$
\dot{\mathbf{s}}_{2}=\mathbf{I}^{-1} \dot{\mathbf{u}}_{s}+\Delta \dot{\mathbf{D}}_{2}
$$

Based on Assumption 1, the following assumption holds.

Assumption 5. The term $\Delta \dot{\mathbf{D}}_{2}$ satisfies $\left\|\Delta \dot{\mathbf{D}}_{2}\right\| \leq \rho_{2}$, where $\rho_{2}$ is an unknown positive constant.

Since $\rho_{2}$ needs to be estimated online, the representation $\widehat{\rho}_{2}$ will be applied to denote the estimation, and an adaptive law is used to update $\widehat{\rho}_{2}$.

From (29), the nonlinear control term $\mathbf{u}_{s}$ can be designed as

$$
\mathbf{u}_{s}=-\left(\widehat{\rho}_{2}+\varepsilon_{2}\right) \mathbf{I} \int \operatorname{sgn}\left(\mathbf{s}_{2}\right) d t
$$


and the adaptive law for $\widehat{\rho}_{2}$ is

$$
\dot{\hat{\rho}}_{2}=\mu_{2}\left\|\mathbf{s}_{2}\right\| \text {, }
$$

where $\varepsilon_{2}, \mu_{2}>0$ are parameters to be designed.

The Lyapunov candidate function is constructed as

$$
V_{2}=\frac{1}{2} \mathbf{s}_{2}^{T} \mathbf{s}_{2}+\frac{1}{2 \mu_{2}} \widetilde{\rho}_{2}^{2}+\frac{1}{2} \sum_{j=1}^{2} \mathbf{e}_{f j}^{T} \mathbf{e}_{f j},
$$

where $\widetilde{\rho}_{2}$ is the estimation error of $\rho_{2}, \widetilde{\rho}_{2}=\widehat{\rho}_{2}-\rho_{2}$.

The time derivative of $V_{2}$ is

$$
\dot{V}_{2}=\mathbf{s}_{2}^{T} \dot{\mathbf{s}}_{2}+\frac{1}{\mu_{2}} \tilde{\rho}_{2} \dot{\hat{\rho}}_{2}+\sum_{j=1}^{2} \mathbf{e}_{f j}^{T} \dot{\mathbf{e}}_{f j} .
$$

The last term $\sum_{j=1}^{2} \mathbf{e}_{f j}^{T} \dot{\mathbf{e}}_{f j}$ satisfies the following inequality [50]:

$$
\begin{aligned}
\sum_{j=1}^{2} \mathbf{e}_{f j}^{T} \dot{\mathbf{e}}_{f j} \leq & -\left\|\left(\widehat{\boldsymbol{\omega}}_{d}-\boldsymbol{\omega}_{d}\right)^{T}\right\| c_{1} \\
& \cdot\left(\frac{\bar{\psi}_{1}\left\|\widehat{\boldsymbol{\omega}}_{d}-\boldsymbol{\omega}_{d}\right\|}{\left\|\widehat{\boldsymbol{\omega}}_{d}-\boldsymbol{\omega}_{d}\right\|+\sigma_{1}}-1\right)-\left\|\left(\overline{\boldsymbol{\omega}}_{d}-\dot{\overline{\boldsymbol{\omega}}}_{d}\right)^{T}\right\| c_{2} \\
& \cdot\left(\frac{\bar{\psi}_{2}\left\|\overline{\boldsymbol{\omega}}_{d}-\dot{\widehat{\boldsymbol{\omega}}}_{d}\right\|}{\left\|\overline{\boldsymbol{\omega}}_{d}-\dot{\overline{\boldsymbol{\omega}}}_{d}\right\|+\sigma_{2}}-1\right)
\end{aligned}
$$

with the following assumptions: (1) the time derivative of $\boldsymbol{\omega}_{d}$ is bounded with a known positive constant $c_{1}$ and satisfies $\left\|\dot{\boldsymbol{\omega}}_{d}\right\| \leq\left\|\dot{\boldsymbol{\omega}}_{d}\right\|_{\max } \leq c_{1}$, and $\psi_{1}=\bar{\psi}_{1} c_{1}, \bar{\psi}_{1}>1$. (2) $\dot{\overline{\boldsymbol{\omega}}}_{d}$ is bounded with a constant $c_{2}>0$ and satisfies $\left\|\dot{\vec{\omega}}_{d}\right\| \leq$ $\left\|\dot{\vec{\omega}}_{d}\right\|_{\max } \leq c_{2}$ and $\psi_{2}=\bar{\psi}_{2} c_{2}, \bar{\psi}_{2}>1$.

If $\left\|\widehat{\boldsymbol{\omega}}_{d}-\boldsymbol{\omega}_{d}\right\|>\sigma_{1} /\left(\bar{\psi}_{1}-1\right)$ and $\left\|\overline{\boldsymbol{\omega}}_{d}-\dot{\overline{\boldsymbol{\omega}}}_{d}\right\|>\sigma_{2} /\left(\bar{\psi}_{2}-1\right)$, we obtain

$$
\sum_{j=1}^{2} \mathbf{e}_{f j}^{T} \dot{\mathbf{e}}_{f j} \leq 0 .
$$

The convergence of the filter is assured, and the estimation error can be guaranteed within the compact set determined in the following form [50]: $\left\|\widehat{\boldsymbol{\omega}}_{d}-\boldsymbol{\omega}_{d}\right\| \leq \sigma_{1} /\left(\bar{\psi}_{1}-1\right),\left\|\overline{\boldsymbol{\omega}}_{d}-\dot{\overline{\boldsymbol{\omega}}}_{d}\right\| \leq$ $\sigma_{2} /\left(\bar{\psi}_{2}-1\right)$. The estimation error of the filter can be adjusted sufficiently small by choosing appropriate values of $\sigma_{1}, \sigma_{2}$.

Based on (29)-(31), and (35), the equality (33) satisfies

$$
\begin{aligned}
\dot{V}_{2} & \leq \mathbf{s}_{2}^{T}\left[-\left(\hat{\rho}_{2}+\varepsilon_{2}\right) \mathbf{I} \operatorname{sgn}\left(\mathbf{s}_{2}\right)+\Delta \dot{\mathbf{D}}\right]+\hat{\rho}_{2}\left\|\mathbf{s}_{2}\right\|-\rho_{2}\left\|\mathbf{s}_{2}\right\| \\
& \leq-\varepsilon_{2}\left\|\mathbf{s}_{2}\right\| .
\end{aligned}
$$

If $\left\|\mathbf{s}_{2}\right\| \neq 0$, then $\dot{V}_{2}<0$, and $\mathbf{s}_{2}$ is asymptotically stable. Through the similar analysis shown in Section 3.1, the following inequality holds:

$$
\left\|\dot{\mathbf{s}}_{2}\right\| \geq \varepsilon_{2}+\tilde{\rho}_{2}>0 .
$$

It indicates that $\mathbf{s}_{2}$ will converge to origin in finite time, after it reaches the surface $\mathbf{s}_{2}=0$, and $\mathbf{z}_{2}$ and $\dot{\mathbf{z}}_{2}$ will reach the origin in finite time. So $\mathbf{z}_{2}$ converges to origin in finite time; then the virtual control input $\boldsymbol{\omega}_{d}$ will force $\mathbf{z}_{1}$ to converge to origin in finite time; that is, the guidance command of attitude angle is stably tracked in finite time.

Remark 6. There are the integrals of the discontinuous signals in (18) and (30). Thus, the control input is continuous and the chattering caused by the sign function is alleviated by the integration.

\section{Constrained Adaptive Backstepping Fast Terminal Sliding Mode Control (CABFTSMC) Design}

It is unavoidable that actuator is limited in the control system of hypersonic vehicles, especially the magnitude constraints of actuator inputs. So in this section, we will further study the controller design taking input constraint, model uncertainty and external disturbance into account.

The control objective is to design controller for the COM (5)-(6) to guarantee that the output $\Omega$ tracks the guidance command $\Omega_{d}$, even in spite of input constraint, model uncertainty, and external disturbance, with the assumption that every element of $\boldsymbol{\Omega}_{d}$ and its first order time derivative are continuous and bounded.

The virtual control input and adaptive law for the attitude angle subsystem (5) are (13) and (19) developed in Section 3. In what follows, the control input is designed with the consideration of input constraint, model uncertainty, and external disturbance.

Considering input constraint $\mathbf{u}=\operatorname{sat}\left(\mathbf{u}_{0}\right)$, the attitude angular rate dynamic (6) can be rewritten as

$$
\dot{\omega}=-\mathbf{I}^{-1} \Phi \mathrm{I} \boldsymbol{\omega}+\mathbf{I}^{-1} \text { sat }\left(\mathbf{u}_{0}\right)+\Delta \mathrm{D} .
$$

Here, $\mathbf{u}$ is the actual control generated by the actuator, and $\operatorname{sat}\left(\mathbf{u}_{0}\right)$ denotes the nonlinear saturation characteristic.

To handle input constraint conveniently, the saturation function $\operatorname{sat}\left(\mathbf{u}_{0}\right)$ can be expressed as

$$
\operatorname{sat}\left(\mathbf{u}_{0}\right)=\boldsymbol{\delta}_{u_{0}} \mathbf{u}_{0} .
$$

Here, $\operatorname{sat}\left(\mathbf{u}_{0}\right)=\left[\begin{array}{lll}\operatorname{sat}\left(u_{01}\right) & \operatorname{sat}\left(u_{02}\right) & \operatorname{sat}\left(u_{03}\right)\end{array}\right]^{T}$, and $\operatorname{sat}\left(u_{0 i}\right)$ is the following formulation:

$$
\operatorname{sat}\left(u_{0 i}\right)= \begin{cases}u_{i \max }, & u_{0 i} \geq u_{i \max } \\ u_{0 i}, & -u_{i \max }<u_{0 i}<u_{i \max } \\ -u_{i \max }, & u_{0 i} \leq-u_{i \max } .\end{cases}
$$

For simplicity, we assume that three control inputs have the same bound; that is, $u_{i \max }=\bar{u}_{\max }$. Motivated by the research in reference [51], the following assumption holds.

Assumption 7. The relationship between $\bar{u}_{\max }$ and $\bar{d}_{1}$ satisfies the following inequality: $\bar{u}_{\max }>\bar{d}_{1}$.

To proceed, from (39), we obtain

$$
\operatorname{sat}\left(u_{0 i}\right)=\delta_{u_{0 i}} u_{0 i} \text {. }
$$


Here, $\delta_{u_{0 i}}$ has the following expression:

$$
\delta_{u_{0 i}}= \begin{cases}\frac{u_{i_{\max }}}{u_{0 i}}, & u_{0 i} \geq u_{i_{\max }} \\ 1, & -u_{i_{\max }}<u_{0 i}<u_{i_{\max }} \\ -\frac{u_{i_{\max }}}{u_{0 i}}, & u_{0 i} \leq-u_{i_{\max }} .\end{cases}
$$

Note that $0<\delta_{u_{0 i}} \leq 1$, and it can be regarded as an indicator of the saturation degree of the control input $u_{0 i}$; if $\delta_{u_{0 i}}$ approaches zero, there is almost no feedback from input $u_{0 i}$, while $\delta_{u_{0 i}}=1$ means that $u_{0 i}$ does not saturate. According to the density property of a real number [52], there is a constant $r_{u_{0 i}}$ which satisfies

$$
0<r_{u_{0 i}} \leq \delta_{u_{0 i}} \leq 1
$$

From (39), (38) can be rewritten as

$$
\dot{\omega}=-\mathbf{I}^{-1} \Phi \mathbf{I} \boldsymbol{\omega}+\mathbf{I}^{-1} \delta_{\mathbf{u}_{0}} \mathbf{u}_{0}+\Delta \mathbf{D}_{2}
$$

Taking time derivative of (11) along with (44), we have

$$
\dot{\mathbf{z}}_{2}=-\mathbf{I}^{-1} \Phi \mathbf{I} \boldsymbol{\omega}+\mathbf{I}^{-1} \boldsymbol{\delta}_{\mathbf{u}_{0}} \mathbf{u}_{0}+\Delta \mathbf{D}_{2}-\dot{\boldsymbol{\omega}}_{d}
$$

For the term $\Delta \mathrm{D}_{2}-\dot{\boldsymbol{\omega}}_{d}$, the following assumption holds.

Assumption 8. There is a positive constant $\rho_{3}$ which makes the following condition hold: $\left\|\Delta \mathbf{D}_{2}-\dot{\boldsymbol{\omega}}_{d}\right\| \leq \rho_{3}$.

Since $\rho_{3}$ needs to be estimated online, the representation $\widehat{\rho}_{3}$ will be applied to denote the estimation, and an adaptive law is used to update $\widehat{\rho}_{3}$.

The control input $\mathbf{u}_{0}$ is designed as

$$
\mathbf{u}_{0}=-\|\mathbf{I}\|^{2} \lambda_{5} \mathbf{I}^{-T} \mathbf{z}_{2}-\frac{\widehat{r}_{\mathbf{u}_{0}}\left(\left\|\mathbf{I}^{-1} \mathbf{\Phi} \mathbf{I} \boldsymbol{\omega}\right\|+\hat{\rho}_{3}+\varepsilon_{3}\right) \mathbf{I} \mathbf{z}_{2}}{\left\|\mathbf{z}_{2}\right\|},
$$

and the adaptive laws for $\widehat{\rho}_{3}$ and $\widehat{r}_{\mathbf{u}_{0}}$ are

$$
\begin{gathered}
\dot{\hat{\rho}}_{3}=\mu_{3}\left\|\mathbf{z}_{2}\right\|, \\
\dot{\hat{r}}_{\mathbf{u}_{0}}=\mu_{4}\left(\left\|\mathbf{I}^{-1} \mathbf{\Phi} \mathbf{I} \boldsymbol{\omega}\right\|+\widehat{\rho}_{3}\right) \widehat{r}_{\mathbf{u}_{0}}^{3}\left\|\mathbf{z}_{2}\right\|,
\end{gathered}
$$

where $\lambda_{5}, \varepsilon_{3}, \mu_{3}, \mu_{4}, \widehat{r}_{\mathbf{u}_{0}}(0), \widehat{\rho}_{3}(0)>0$.

The Lyapunov function is constructed as

$$
V_{3}=\frac{1}{2} \mathbf{z}_{2}^{T} \mathbf{z}_{2}+\frac{1}{2 \mu_{3}} \widetilde{\rho}_{3}^{2}+\frac{1}{2 \mu_{4}} \widetilde{r}_{\mathbf{u}_{0}}^{2},
$$

where $\widetilde{\rho}_{3}=\widehat{\rho}_{3}-\rho_{3}$ is the estimation error of $\rho_{3}$, and $\widetilde{r}_{\mathbf{u}_{0}}=$ $\widehat{r}_{\mathbf{u}_{0}}^{-1}-r_{\mathbf{u}_{0}}$.

Using the derivative of $V_{3}$ with respect to time, then

$$
\dot{V}_{3}=\mathbf{z}_{2}^{T} \dot{\mathbf{z}}_{2}+\frac{1}{\mu_{3}} \widetilde{\rho}_{3} \dot{\hat{\rho}}_{3}+\frac{1}{\mu_{4}} \widetilde{r}_{\mathbf{u}_{0}} \widehat{r}_{\mathbf{u}_{0}}^{-2} \dot{\widehat{r}}_{\mathbf{u}_{0}} \cdot
$$

Based on (46) and (47), the above equation satisfies

$$
\begin{aligned}
& \dot{V}_{3}=\mathbf{z}_{2}^{T}\left(-\mathbf{I}^{-1} \boldsymbol{\Phi} \mathbf{I} \boldsymbol{\omega}+\mathbf{I}^{-1} \boldsymbol{\delta}_{\mathbf{u}_{0}} \mathbf{u}_{0}+\Delta \mathbf{D}-\dot{\boldsymbol{\omega}}_{d}\right) \\
& +\widetilde{\rho}_{3}\left\|\mathbf{z}_{2}\right\|-\widetilde{r}_{\mathbf{u}_{0}} \widehat{r}_{\mathbf{u}_{0}}\left(\left\|\mathbf{I}^{-1} \boldsymbol{\Phi} \mathbf{I} \boldsymbol{\omega}\right\|+\widehat{\rho}_{3}\right)\left\|\mathbf{z}_{2}\right\| \\
& \leq\left\|\mathbf{z}_{2}\right\|\left\|\mathbf{I}^{-1} \boldsymbol{\Phi} \mathbf{I} \boldsymbol{\omega}\right\|-\widehat{r}_{\mathbf{u}_{0}}\left\|\mathbf{z}_{2}\right\| \delta_{\mathbf{u}_{0}} \\
& \cdot\left(\left\|\mathbf{I}^{-1} \boldsymbol{\Phi} \mathbf{I} \boldsymbol{\omega}\right\|+\widehat{\rho}_{3}+\varepsilon_{3}\right)-\lambda_{5} \boldsymbol{\delta}_{\mathbf{u}_{0}}\left\|\mathbf{z}_{2}\right\|^{2} \\
& +\left\|\mathbf{z}_{2}\right\| \rho_{3}+\left\|\mathbf{z}_{2}\right\| \tilde{\rho}_{3}-\widetilde{r}_{\mathbf{u}_{0}} \widehat{r}_{\mathbf{u}_{0}}\left(\left\|\mathbf{I}^{-1} \boldsymbol{\Phi} \mathbf{I} \boldsymbol{\omega}\right\|+\widehat{\rho}_{3}\right)\left\|\mathbf{z}_{2}\right\| \\
& \leq\left\|\mathbf{z}_{2}\right\|\left(\left\|\mathbf{I}^{-1} \boldsymbol{\Phi} \mathbf{I} \boldsymbol{\omega}\right\|+\rho_{3}\right)-\lambda_{5} \boldsymbol{\delta}_{\mathbf{u}_{0}}\left\|\mathbf{z}_{2}\right\|^{2} \\
& -\left\|\mathbf{z}_{2}\right\| \boldsymbol{\delta}_{\mathbf{u}_{0}} \widehat{r}_{\mathbf{u}_{0}}\left(\left\|\mathbf{I}^{-1} \boldsymbol{\Phi} \mathbf{I} \boldsymbol{\omega}\right\|+\widehat{\rho}_{3}\right) \\
& -\left\|\mathbf{z}_{2}\right\| \boldsymbol{\delta}_{\mathbf{u}_{0}} \widehat{r}_{\mathbf{u}_{0}} \varepsilon_{3}+\tilde{\rho}_{3}\left\|\mathbf{z}_{2}\right\| \\
& -\left\|\mathbf{z}_{2}\right\|\left(\left\|\mathbf{I}^{-1} \boldsymbol{\Phi} \mathbf{I} \boldsymbol{\omega}\right\|+\widehat{\rho}_{3}\right) \\
& +r_{\mathbf{u}_{0}} \widehat{r}_{\mathbf{u}_{0}}\left(\left\|\mathbf{I}^{-1} \boldsymbol{\Phi} \mathbf{I} \boldsymbol{\omega}\right\|+\widehat{\rho}_{3}\right)\left\|\mathbf{z}_{2}\right\| \\
& =-\lambda_{5} \boldsymbol{\delta}_{\mathbf{u}_{0}}\left\|\mathbf{z}_{2}\right\|^{2}-\delta_{\mathbf{u}_{0}} \widehat{r}_{\mathbf{u}_{0}} \\
& \cdot\left(\left\|\mathbf{I}^{-1} \boldsymbol{\Phi} \mathbf{I} \boldsymbol{\omega}\right\|+\widehat{\rho}_{3}\right)\left\|\mathbf{z}_{2}\right\|-\left\|\mathbf{z}_{2}\right\| \boldsymbol{\delta}_{\mathbf{u}_{0}} \widehat{r}_{\mathbf{u}_{0}} \varepsilon_{3} \\
& +r_{\mathbf{u}_{0}} \widehat{r}_{\mathbf{u}_{0}}\left(\left\|\mathbf{I}^{-1} \boldsymbol{\Phi} \mathbf{I} \boldsymbol{\omega}\right\|+\widehat{\rho}_{3}\right)\left\|\mathbf{z}_{2}\right\| \\
& \leq-\lambda_{5} \boldsymbol{\delta}_{\mathbf{u}_{0}}\left\|\mathbf{z}_{2}\right\|^{2}-\boldsymbol{\delta}_{\mathbf{u}_{0}} \widehat{r}_{\mathbf{u}_{0}}\left\|\mathbf{z}_{2}\right\| \varepsilon_{3} \\
& \leq-\lambda_{\min }\left(\lambda_{5} \boldsymbol{\delta}_{\mathbf{u}_{0}}\right)\left\|\mathbf{z}_{2}\right\|^{2}-\left\|\mathbf{z}_{2}\right\| \varepsilon_{3} .
\end{aligned}
$$

By integrating (47), $\widehat{\rho}_{3}(t)=\widehat{\rho}_{3}(0)+\mu_{3} \int_{0}^{t}\left\|\mathbf{z}_{2}\right\| d t, \widehat{r}_{\mathbf{u}_{0}}(t)=$ $\widehat{r}_{\mathbf{u}_{0}}(0)+\mu_{4} \int_{0}^{t}\left(\left\|\mathbf{I}^{-1} \boldsymbol{\Phi} \mathbf{I} \boldsymbol{\omega}\right\|+\widehat{\rho}_{3}\right) \widehat{r}_{\mathbf{u}_{0}}^{3}\left\|\mathbf{z}_{2}\right\| d t$. So when $\left\|\mathbf{z}_{2}\right\| \neq 0$, if the values $\widehat{\rho}_{3}(0), \widehat{r}_{\mathbf{u}_{0}}(0), \mu_{3}$, and $\mu_{4}$ are chosen appropriately, then there is a time $t_{2}$ to make $\widehat{\rho}_{3}(t) \geq \rho_{3}(t)$ and $\widehat{r}_{\mathbf{u}_{0}}(t) \geq \widehat{r}_{\mathbf{u}_{0}}^{-1}(t)$ hold for any random time $t>t_{2}$. Moreover, $\tilde{\rho}_{3}(t) \geq 0$ and $\tilde{r}_{\mathbf{u}_{0}} \leq 0$.

From (50), $\mathbf{z}_{2}^{T} \dot{\mathbf{z}}_{2}+\tilde{\rho}_{3}\left\|\mathbf{z}_{2}\right\|-\widetilde{r}_{\mathbf{u}_{0}} \widehat{r}_{\mathbf{u}_{0}}\left(\left\|\mathbf{I}^{-1} \boldsymbol{\Phi} \mathbf{I} \boldsymbol{\omega}\right\|+\widehat{\rho}_{3}\right)\left\|\mathbf{z}_{2}\right\| \leq$ $-\varepsilon_{3}\left\|\mathbf{z}_{2}\right\|$ as long as $t>t_{2}$, and the following inequality holds:

$$
\begin{aligned}
\left\|\dot{\mathbf{z}}_{2}\right\| \geq & \left(\widetilde{\rho}_{3}+\varepsilon_{3}\right)\left\|\mathbf{z}_{2}\right\| \\
& -\widetilde{r}_{\mathbf{u}_{0}} \widehat{r}_{\mathbf{u}_{0}}\left(\left\|-\mathbf{I}^{-1} \Phi \mathbf{I} \boldsymbol{\omega}\right\|+\widehat{\rho}_{3}\right)\left\|\mathbf{z}_{2}\right\|>0 .
\end{aligned}
$$

It is obvious that $\mathbf{z}_{2}$ will converge to origin in finite time. Then, $\mathbf{z}_{1}$ will converge to the origin in finite time under the influence of virtual control input (13). 
Remark 9. The CABFTSMC law (46) is discontinuous when crossing $\mathbf{z}_{2}$ which may result in undesirable chattering. It can be alleviated by introducing a so-called boundary layer around $\mathbf{z}_{2}$ [53]. Thus, the controller (46) is revised as

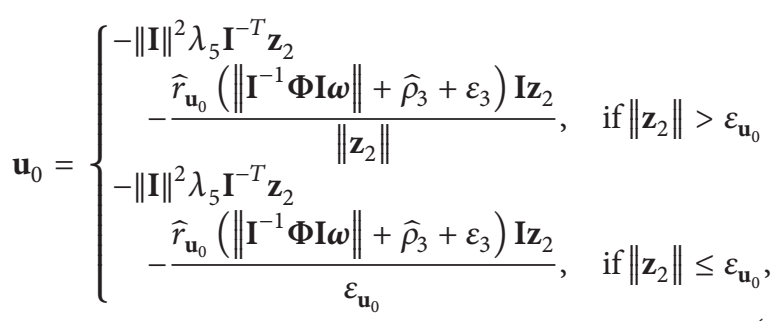

where $\varepsilon_{\mathbf{u}_{0}}$ is a positive constant.

Remark 10. At the control design level, $\dot{\mathbf{z}}_{1}$ and $\dot{\mathbf{z}}_{2}$ are utilized. It is noted that the uncertainty of (5) and (6) may induce that they cannot be computed. So, the following robust differentiators are employed to estimate $\dot{\mathbf{z}}_{1}$ and $\dot{\mathbf{z}}_{2}$ [54]:

$$
\begin{array}{r}
\dot{\mathbf{w}}_{0, j}=-k_{0, j} \operatorname{abs}\left(\mathbf{w}_{0, j}-\mathbf{z}_{j}\right)^{1 / 2} \operatorname{sign}\left(\mathbf{w}_{0, j}-\mathbf{z}_{j}\right)+\mathbf{w}_{1, j} \\
\dot{\mathbf{w}}_{1, j}=-k_{1, j} \operatorname{abs}\left(\mathbf{w}_{1, j}-\mathbf{w}_{0, j}\right)^{1 / 2} \operatorname{sign}\left(\mathbf{w}_{1, j}-\mathbf{w}_{0, j}\right), \\
j=1,2 .
\end{array}
$$

To alleviate the chattering in the robust differentiators (53), a saturation function sat $(\cdot)$ is generally employed to replace the sign function $\operatorname{sign}(\cdot)$ as follows:

$$
\begin{aligned}
& \dot{w}_{0, j i}=-k_{0, j} \operatorname{abs}\left(w_{0, j i}-z_{j i}\right)^{1 / 2} \operatorname{sat}\left(w_{0, j i}-z_{j i}\right)+w_{1, j i} \\
& \dot{w}_{1, j i}=-k_{1, j} \operatorname{abs}\left(w_{1, j i}-w_{0, j i}\right)^{1 / 2} \operatorname{sat}\left(w_{1, j i}-w_{0, j i}\right), \\
& i=1,2,3 \text {, } \\
& \operatorname{sat}\left(w_{0, j i}-z_{j i}\right) \\
& =\left\{\begin{array}{l}
\frac{\left(w_{0, j i}-z_{j i}\right)}{\varepsilon_{0 i}} \\
\operatorname{sign}\left(\frac{\left(w_{0, j i}-z_{j i}\right)}{\varepsilon_{0 i}}\right)
\end{array}\right. \\
& \begin{array}{r}
\text { if } \frac{\left|w_{0, j i}-z_{j i}\right|}{\varepsilon_{0 i}} \leq 1 \\
\text { if } \frac{\left|w_{0, j i}-z_{j i}\right|}{\varepsilon_{0 i}}>1, \\
j=1,2,
\end{array} \\
& \operatorname{sat}\left(w_{1, j i}-w_{0, j i}\right) \\
& = \begin{cases}\frac{\left(w_{1, j i}-w_{0, j i}\right)}{\varepsilon_{1 i}}, & \text { if } \frac{\left|w_{1, j i}-w_{0, j i}\right|}{\varepsilon_{1 i}} \leq 1 \\
\operatorname{sign}\left(\frac{\left(w_{1, j i}-w_{0, j i}\right)}{\varepsilon_{1 i}}\right), & \text { if } \frac{\left|w_{1, j i}-w_{0, j i}\right|}{\varepsilon_{1 i}}>1, \\
j=1,2\end{cases}
\end{aligned}
$$

where $\mathbf{w}_{0, j}=\left[\begin{array}{lll}w_{0, j 1} & w_{0, j 2} & w_{0, j 3}\end{array}\right]^{T}, \mathbf{z}_{j}=\left[\begin{array}{lll}z_{j 1} & z_{j 2} & z_{j 3}\end{array}\right]^{T}$, $\mathbf{w}_{1, j}=\left[\begin{array}{lll}w_{1, j 1} & w_{1, j 2} & w_{1, j 3}\end{array}\right]^{T}$, and $k_{0, j}$ and $k_{1, j}$ are the parameters to be designed.
Remark 11. In practice, $\left\|\mathbf{s}_{1}\right\|,\left\|\mathbf{s}_{2}\right\|$, and $\left\|\mathbf{z}_{2}\right\|$ cannot be zero exactly. Based on (19), (31), and (47), $\widehat{\rho}_{1}, \widehat{\rho}_{2}, \widehat{\rho}_{3}$, and $\widehat{r}_{\mathbf{u}_{0}}$ will increase boundlessly which may cause the boundlessness of the control inputs. In order to avoid this problem, the following revised adaptive laws are used:

$$
\begin{gathered}
\dot{\hat{\rho}}_{1}= \begin{cases}\mu_{1}\left\|\mathbf{s}_{1}\right\|, & \text { if }\left\|\mathbf{s}_{1}\right\|>\varepsilon_{\widehat{\rho}_{1}} \\
0, & \text { if }\left\|\mathbf{s}_{1}\right\| \leq \varepsilon_{\widehat{\rho}_{1}},\end{cases} \\
\dot{\hat{\rho}}_{2}= \begin{cases}\mu_{2}\left\|\mathbf{s}_{2}\right\|, & \text { if }\left\|\mathbf{s}_{2}\right\|>\varepsilon_{\widehat{\rho}_{2}} \\
0, & \text { if }\left\|\mathbf{s}_{2}\right\| \leq \varepsilon_{\widehat{\rho}_{2}},\end{cases} \\
\dot{\hat{\rho}}_{3}= \begin{cases}\mu_{3}\left\|\mathbf{z}_{2}\right\|, & \text { if }\left\|\mathbf{z}_{2}\right\|>\varepsilon_{\mathbf{u}_{0}} \\
0, & \text { if }\left\|\mathbf{z}_{2}\right\| \leq \varepsilon_{\mathbf{u}_{0}},\end{cases} \\
\dot{\hat{r}}_{\mathbf{u}_{0}}= \begin{cases}\mu_{4}\left(\left\|\mathbf{I}^{-1} \Phi \mathbf{I} \boldsymbol{\omega}\right\|+\widehat{\rho}_{3}\right) \widehat{r}_{\mathbf{u}_{0}}^{3}\left\|\mathbf{z}_{2}\right\|, & \text { if }\left\|\mathbf{z}_{2}\right\|>\varepsilon_{\mathbf{u}_{0}} \\
0, & \text { if }\left\|\mathbf{z}_{2}\right\| \leq \varepsilon_{\mathbf{u}_{0}},\end{cases}
\end{gathered}
$$

where $\varepsilon_{\widehat{\rho}_{1}}, \varepsilon_{\widehat{\rho}_{2}}, \varepsilon_{\mathbf{u}_{0}}>0$.

It is worthy to point out that the adaptive laws (56) are utilized to dynamically increase the estimated bounds $\hat{\rho}_{1}, \widehat{\rho}_{2}$, $\widehat{\rho}_{3}$, and $\widehat{r}_{\mathbf{u}_{0}}$ until the corresponding conditions $\left\|\mathbf{s}_{1}\right\| \leq \varepsilon_{\widehat{\rho}_{1}}$, $\left\|\mathbf{s}_{2}\right\| \leq \varepsilon_{\hat{\rho}_{2}}$, and $\left\|\mathbf{z}_{2}\right\| \leq \varepsilon_{\mathbf{u}_{0}}$ are satisfied. As soon as the domains are reached, $\widehat{\rho}_{1}, \widehat{\rho}_{2}, \widehat{\rho}_{3}$, and $\widehat{r}_{\mathbf{u}_{0}}$ stop increasing and keep constant. That is to say, the parameters $\varepsilon_{\widehat{\rho}_{1}}, \varepsilon_{\widehat{\rho}_{2}}$, and $\varepsilon_{\mathbf{u}_{0}}$ are used to avoid the increase of the estimated bounds $\hat{\rho}_{1}$, $\widehat{\rho}_{2}, \widehat{\rho}_{3}$, and $\widehat{r}_{\mathbf{u}_{0}}$ boundlessly. When $\left\|\mathbf{s}_{1}\right\|,\left\|\mathbf{s}_{2}\right\|$, and $\left\|\mathbf{z}_{2}\right\|$ deviate from the domains, the above process will be repeated. It is clear that as $\varepsilon_{\widehat{\rho}_{1}}, \varepsilon_{\widehat{\rho}_{2}}$, and $\varepsilon_{\mathbf{u}_{0}}$ decrease $\widehat{\rho}_{1}, \widehat{\rho}_{2}, \widehat{\rho}_{3}$, and $\widehat{r}_{\mathbf{u}_{0}}$ increase if the other conditions are fixed.

\section{Simulations and Analysis}

In this section, numerical simulations are performed, and the results are shown to present that the proposed control strategies in the previous sections can work effectively. A detailed description of the guidance command is provided in [55]. The normal inertial matrix and the external disturbance vector are given as

$$
\begin{aligned}
\mathbf{I} & =\left[\begin{array}{ccc}
434270 & 0 & -17880 \\
0 & 961200 & 0 \\
-17880 & 0 & 1131541
\end{array}\right], \\
\Delta \mathbf{D}_{1} & =\left[\begin{array}{c}
1+\sin \left(\frac{\pi t}{125}\right)+\sin \left(\frac{\pi t}{250}\right) \\
1+\sin \left(\frac{\pi t}{125}\right)+\sin \left(\frac{\pi t}{250}\right) \\
1+\sin \left(\frac{\pi t}{125}\right)+\sin \left(\frac{\pi t}{250}\right)
\end{array}\right] \times 10^{4} .
\end{aligned}
$$

It is noted that the upper bound of the external disturbance is $\bar{d}_{1}=3 \sqrt{3} \times 10^{4}$. The bound of control authority is assumed to be $\bar{u}_{\max }=6 \times 10^{5}$. Therefore, the control limits used for exploring the capability of the designed control strategy in adhering to the input constraint are $\mathbf{u}_{\max }=(6 \times$ $\left.10^{5} 6 \times 10^{5} 6 \times 10^{5}\right)^{T}$ and $\mathbf{u}_{\min }=-\mathbf{u}_{\max }$. The inertia matrix 

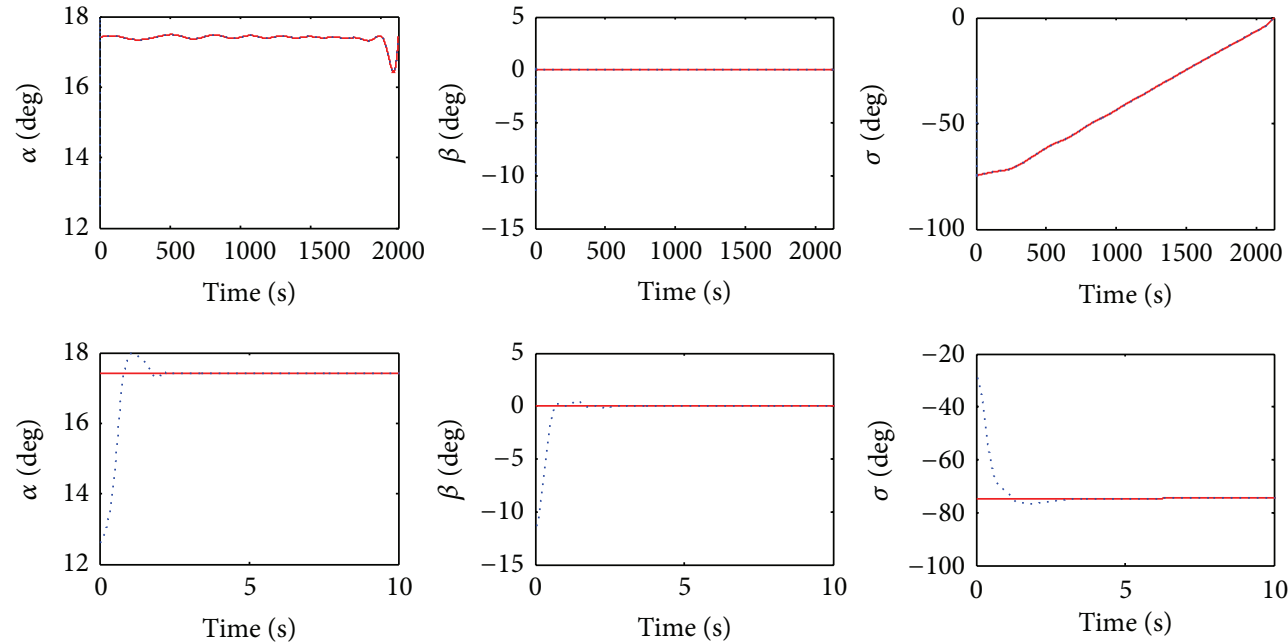

- Guidance command Actual value

- Guidance command Actual value

- Guidance command Actual value

(a) Attitude angle tracking
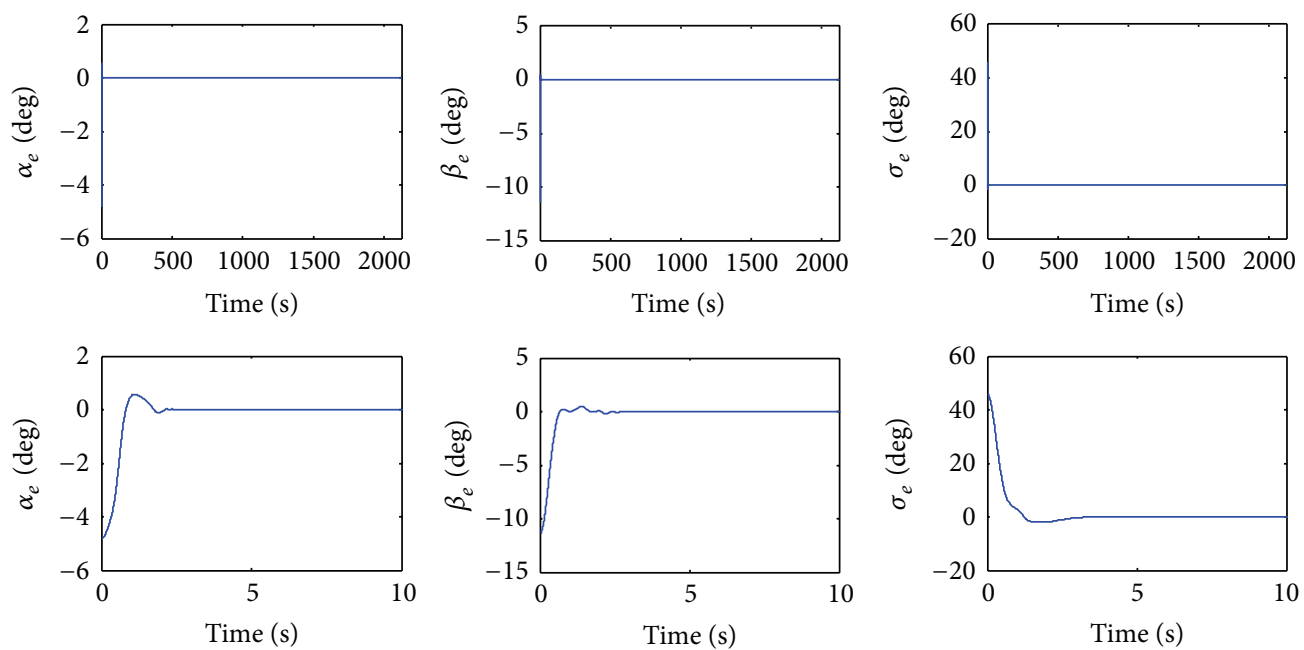

(b) Attitude angle tracking error
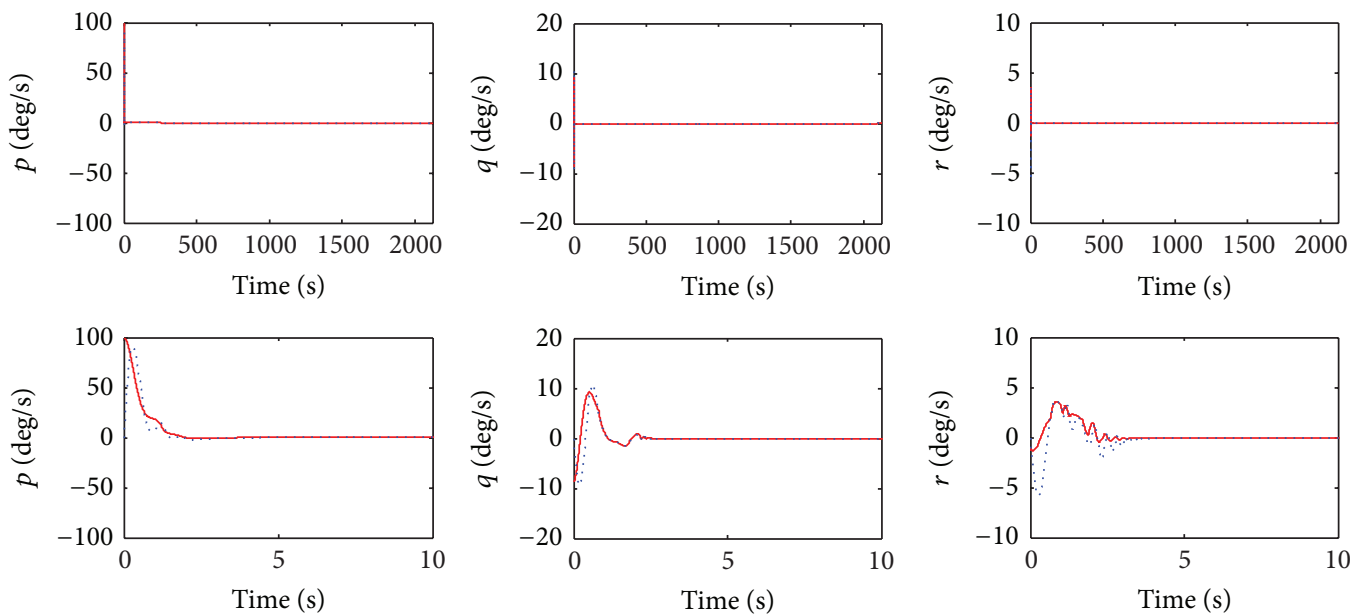

Reference command Actual value

Reference command Actual value

Reference command Actual value

(c) Attitude angular rate tracking

Figure 1: Continued. 

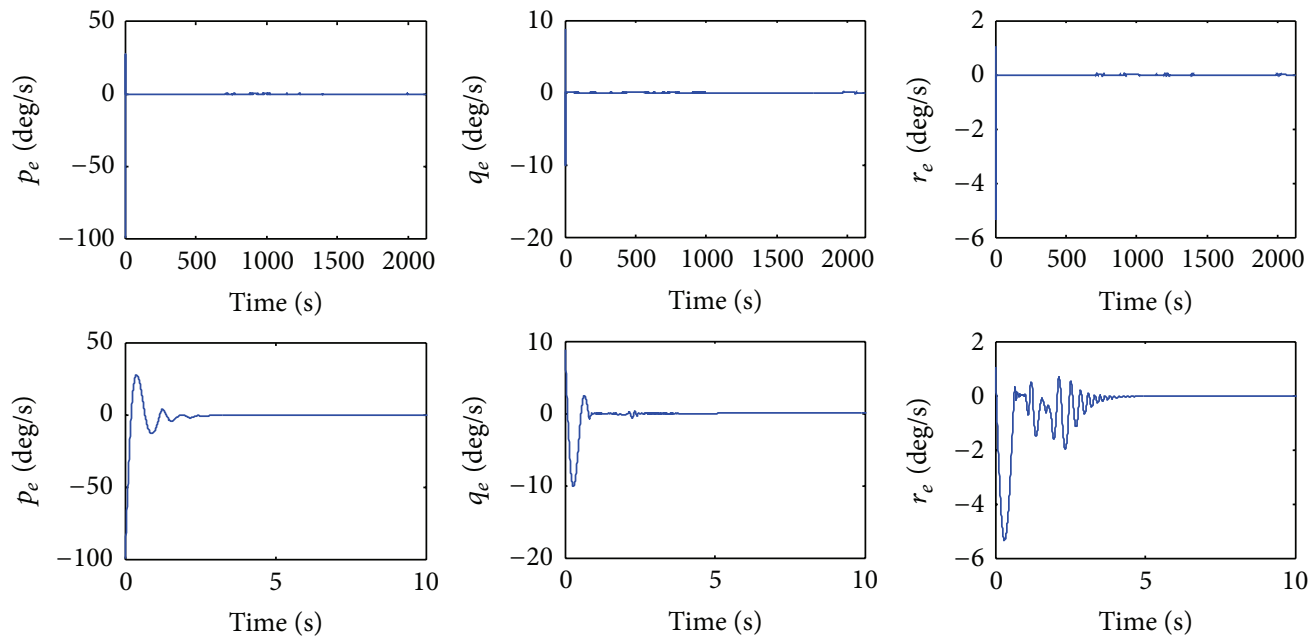

(d) Attitude angular rate tracking error
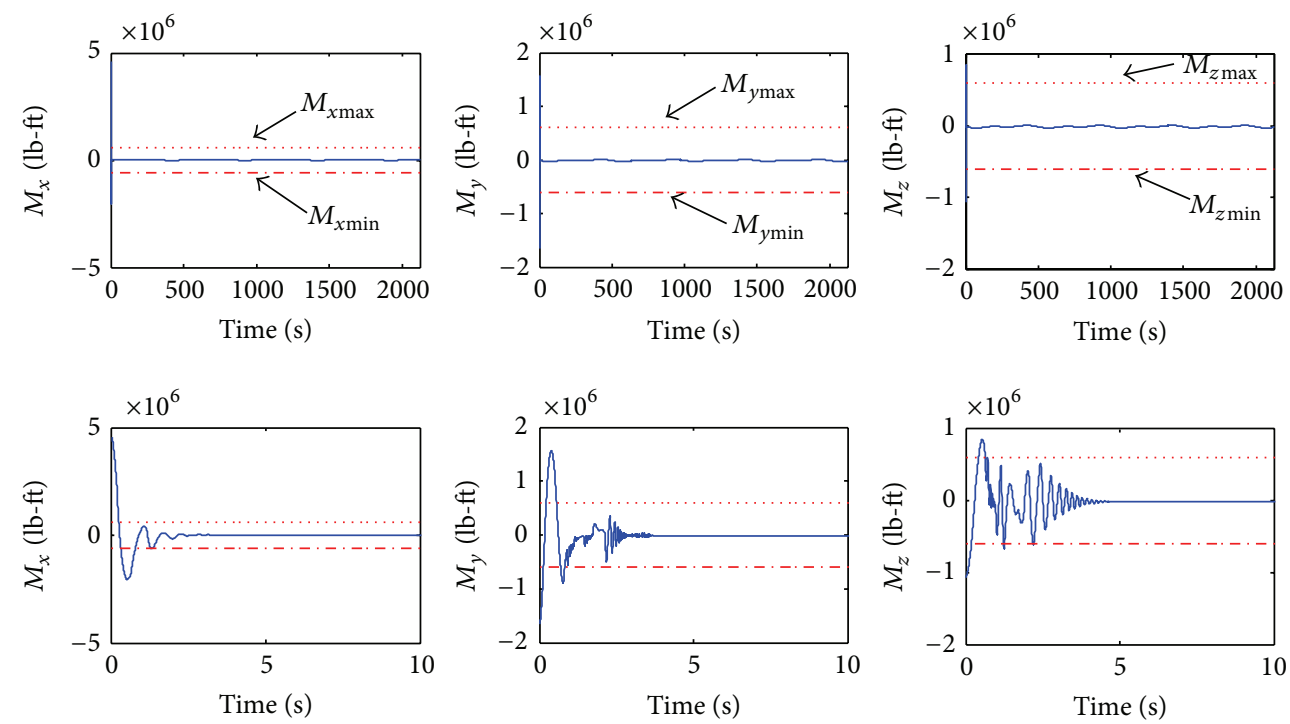

(e) Control inputs

FIgURE 1: Time history of ABFTSMC scheme without input constraint.

uncertainty is assumed to be $\Delta \mathbf{I}=0.2 \mathrm{I}$. The initial condition of reentry flight is given in Table 1 . The controller parameters the control strategies are determined as follows.

The larger parameters $\lambda_{1}, \lambda_{2}, \lambda_{3}$, and $\lambda_{4}$ in (15) and (28) will force the faster convergence rate of the sliding mode surface. In addition, as shown in (21), (36), and (50), larger parameters $\varepsilon_{1}, \varepsilon_{2}, \varepsilon_{3}$, and $\lambda_{5}$ will force the faster convergence rate of the system states. But $\lambda_{1}, \lambda_{2}, \lambda_{3}, \lambda_{4}, \lambda_{5}, \varepsilon_{1}, \varepsilon_{2}$, and $\varepsilon_{3}$ cannot be selected arbitrarily large due to practical considerations such as control input limit. Thus, a compromise is made between the response speed and control input.

The parameters $\mu_{1}, \mu_{2}, \mu_{3}$, and $\mu_{4}$ in (19), (31), and (47) determine the convergence rate of the estimated bounds $\widehat{\rho}_{1}$, $\hat{\rho}_{2}, \hat{\rho}_{3}$ and $\widehat{r}_{\mathbf{u}_{0}}$. We can select $\mu_{1}, \mu_{2}, \mu_{3}$, and $\mu_{4}$ large to guarantee that the estimated bounds $\widehat{\rho}_{1}, \widehat{\rho}_{2}, \widehat{\rho}_{3}$, and $\widehat{r}_{\mathbf{u}_{0}}$ will rapidly converge to the bounds. The large $\hat{\rho}_{1}, \widehat{\rho}_{2}, \widehat{\rho}_{3}$ and $\widehat{r}_{\mathbf{u}_{0}}$ lead to faster accuracy and adjustment ability, but it is at the cost of control chattering or larger control energy. Then the revised adaptive laws (56) are adopted. The parameters $\varepsilon_{\widehat{\rho}_{1}}$, $\varepsilon_{\hat{\rho}_{2}}$, and $\varepsilon_{\mathbf{u}_{0}}$ are utilized to avoid the fact that the estimated bounds $\hat{\rho}_{1}, \hat{\rho}_{2}, \hat{\rho}_{3}$, and $\widehat{r}_{\mathbf{u}_{0}}$ increase boundlessly. The choice of parameters $\varepsilon_{\hat{\rho}_{1}}, \varepsilon_{\hat{\rho}_{2}}$, and $\varepsilon_{\mathbf{u}_{0}}$ is a compromise between the tracking accuracy and control performance.

The parameters $\varepsilon_{0 i}$ and $\varepsilon_{1 i}$ are the widths of boundary layers and employed to deal with the discontinuity in the control law. They are related to the robustness of control scheme. By observing many simulation results, it is appropriate to choose them between $(0,1]$. Moreover, the parameters $\tau_{1}, \tau_{2}, \psi_{1}, \psi_{2}$, $\sigma_{1}$ and $\sigma_{2}$ have influence on the estimation rate of the second order filter (26) and determined by observing the simulated responses in few trials.

Case 1 (simulation results of ABFTSMC with model uncertainty and external disturbance). In this case, the attitude 

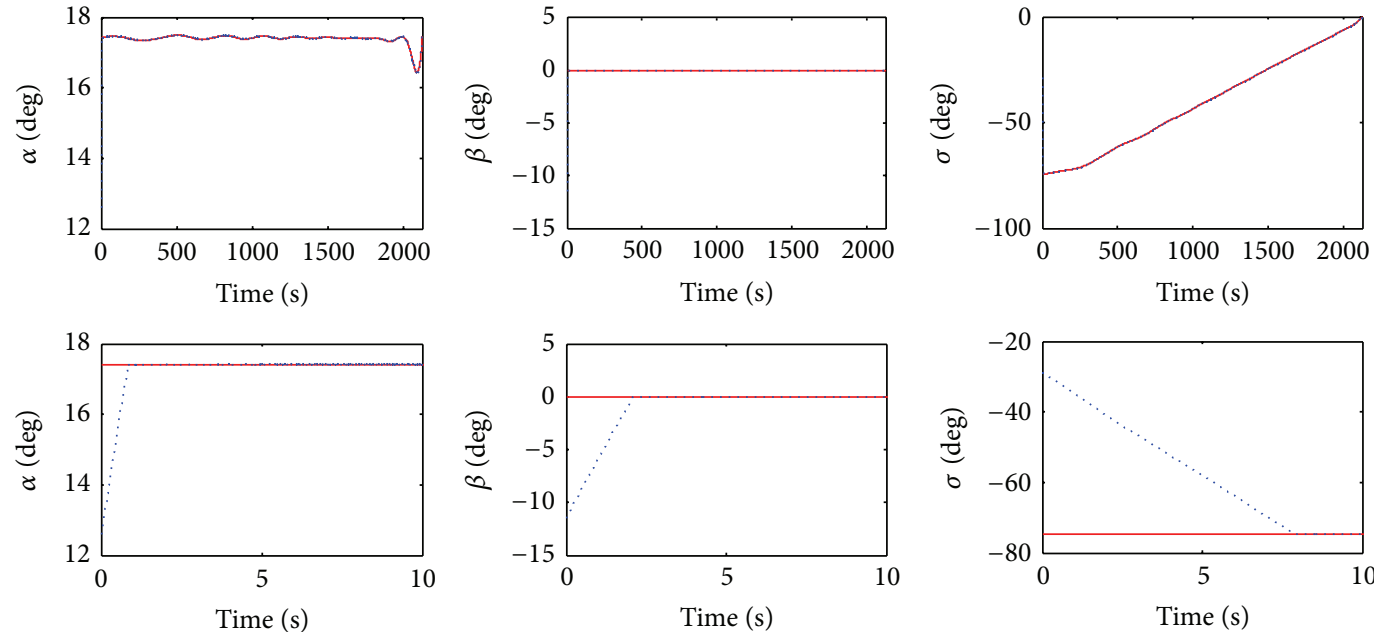

- Guidance command Actual value Actual value

Guidance command Actual value

(a) Attitude angle tracking
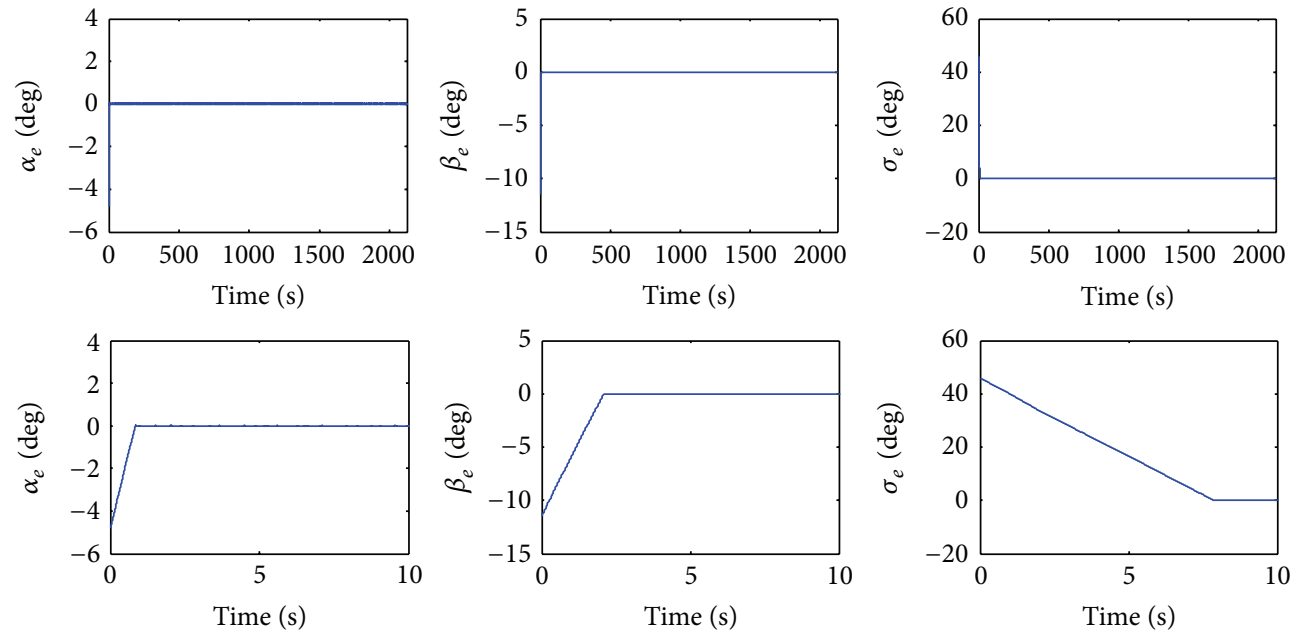

(b) Attitude angle tracking error
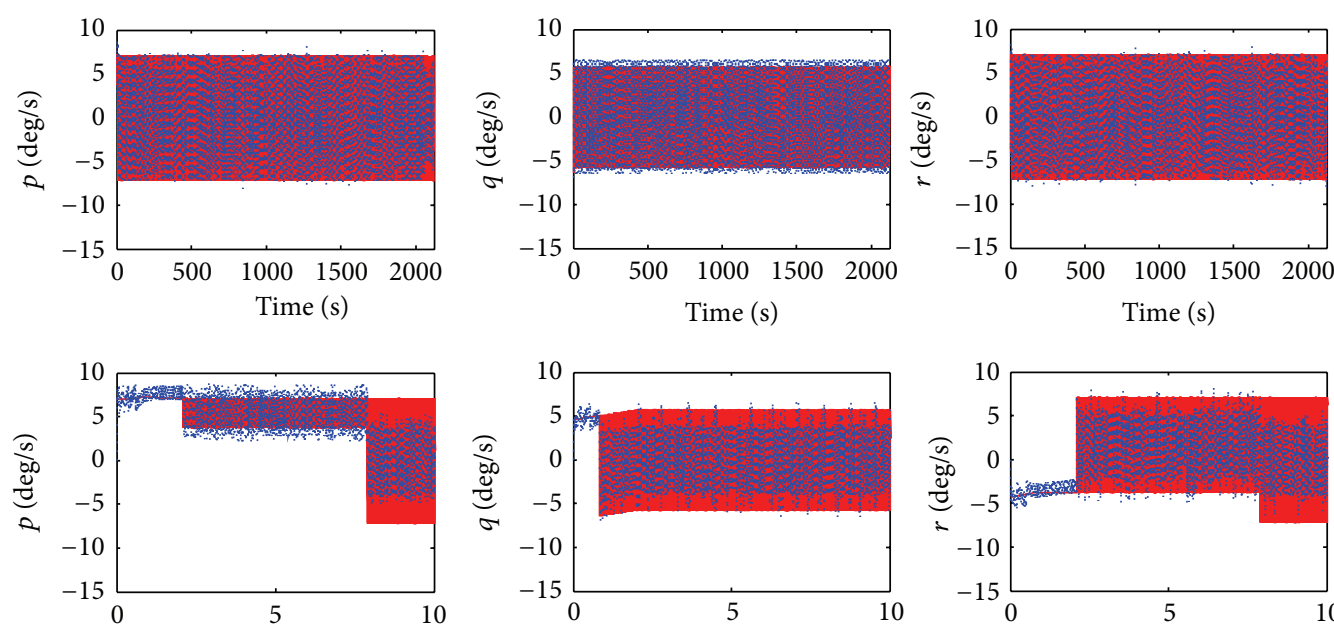

Time (s)
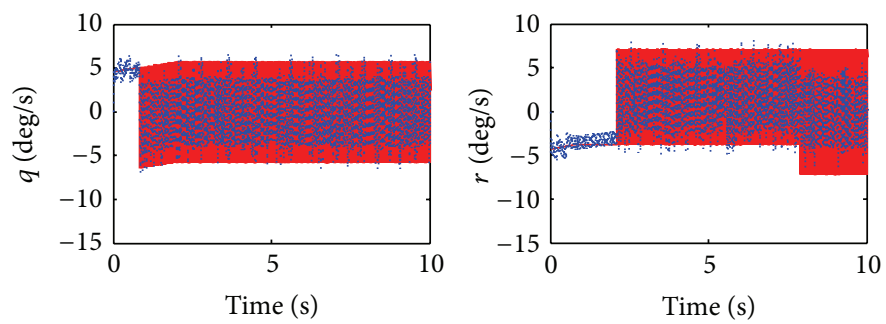

Reference command Actual value

Reference command Actual value

Reference command Actual value

(c) Attitude angular rate tracking

FIgure 2: Continued. 

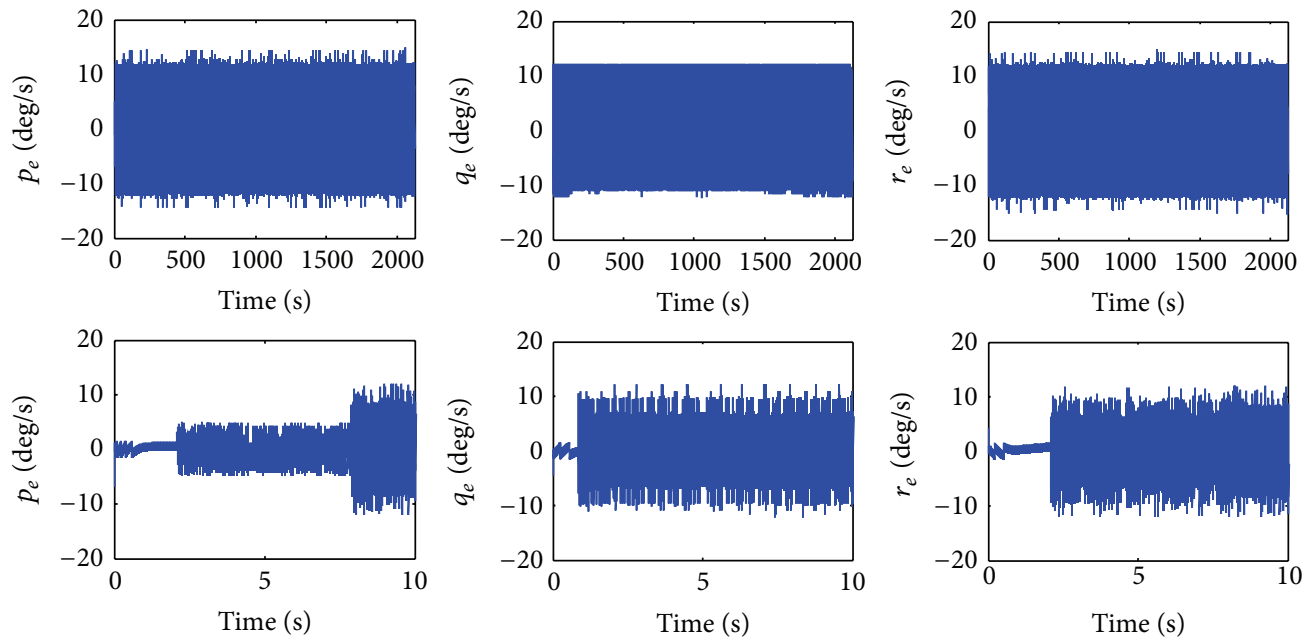

(d) Attitude angular rate tracking error
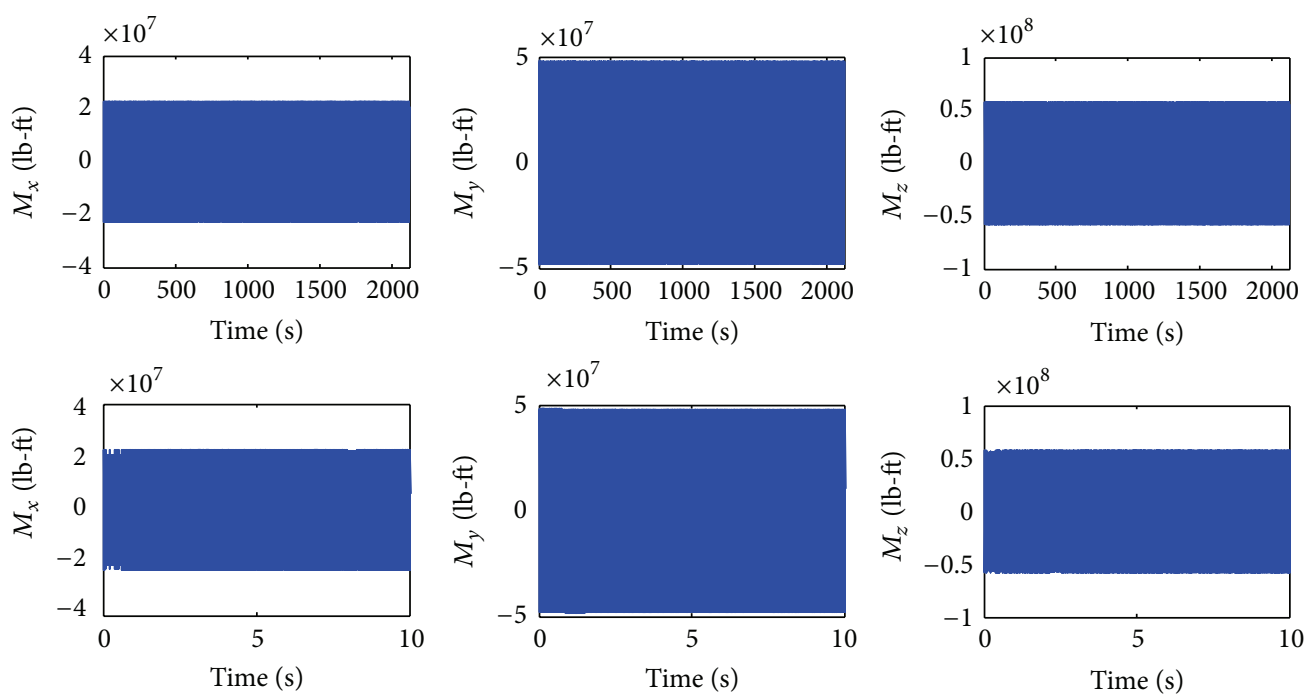

(e) Control inputs

FIGURE 2: Time history of BOSMC scheme without input constraint.

tracking control problem is simulated to demonstrate the effectiveness of controller (24) proposed in Section 3 in handling model uncertainty and external disturbance. The parameters adopted for the proposed control strategy are given in Table 2. Time histories of attitude angle, attitude angular rate, and control inputs are presented in Figure 1. The local time histories are also shown for better demonstration of dynamic process.

Attitude angle tracking performance applying ABFTSMC control strategy is presented in Figures 1(a) and 1(b) which illustrate that the designed control scheme can provide stable tracking of AOA, sideslip angle, and bank angle. They can track their respective guidance commands after about 2.2 seconds, 3.2 seconds, and 3 seconds, respectively. The tracking errors of AOA, sideslip angle, and bank angle converge to zero in finite time despite model uncertainty and external disturbance. As can be seen from Figures 1(c) and $1(\mathrm{~d})$, roll rate, pitch rate, and yaw rate track their reference commands. The tracking errors also keep very small and vanish asymptotically. Although the tracking performance can still be achieved without considering input constraint according to Figures 1(a) and 1(b), control inputs violate their constraints during the transient state process in Figure 1(e). The ABFTSMC scheme without considering input constraint may generate large control signals to attenuate the effect of undesired transient state.

In order to show the chattering alleviation performance of the designed ABFTSMC scheme, the simulation results of the backstepping ordinary sliding mode control (BOSMC) scheme are given for comparison. The controller design procedure is outlined as follows.

A sliding mode manifold for attitude angle subsystem is chosen as $\mathbf{s}_{\Omega}=\mathbf{z}_{1}$, and its time derivative is $\dot{\mathbf{s}}_{\boldsymbol{\Omega}}=\mathbf{R} \boldsymbol{\omega}_{d}+$ $\mathbf{R z}_{2}+\Delta \mathbf{f}_{1}-\dot{\Omega}_{d}$. Based on assumption of the uncertain term 

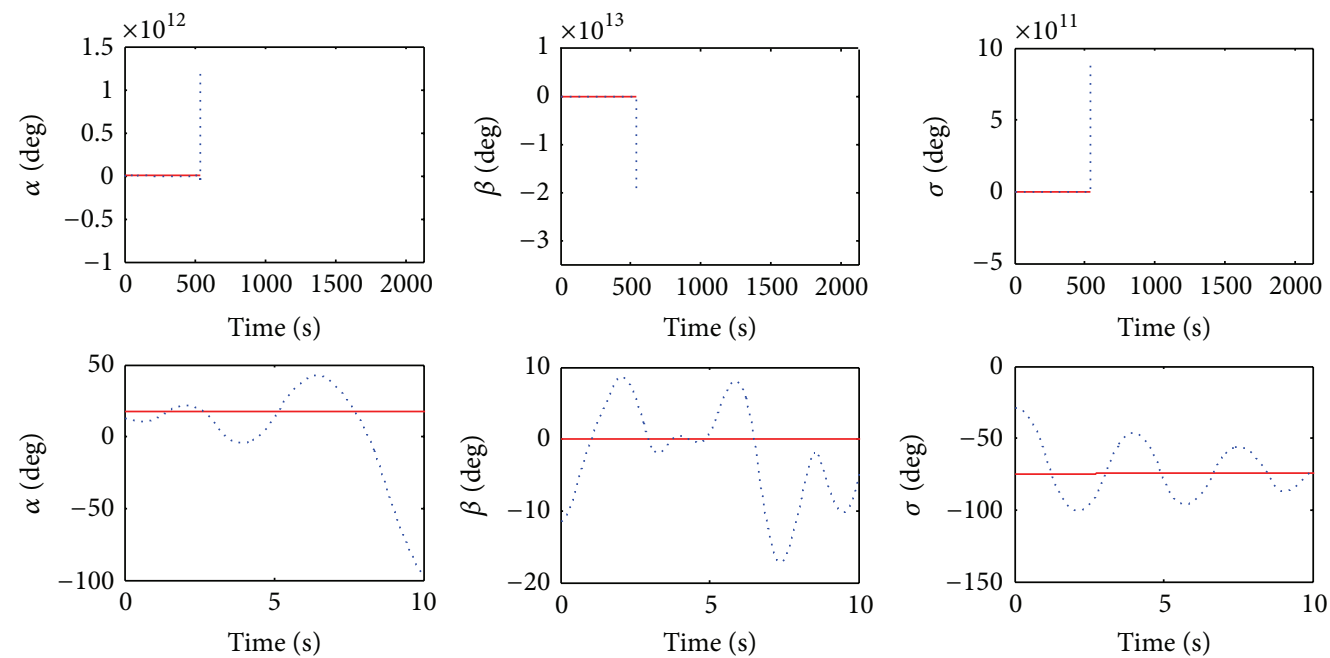

_ Guidance command Actual value

_ Guidance command

Actual value

_ Guidance command

..... Actual value

(a) Attitude angle tracking
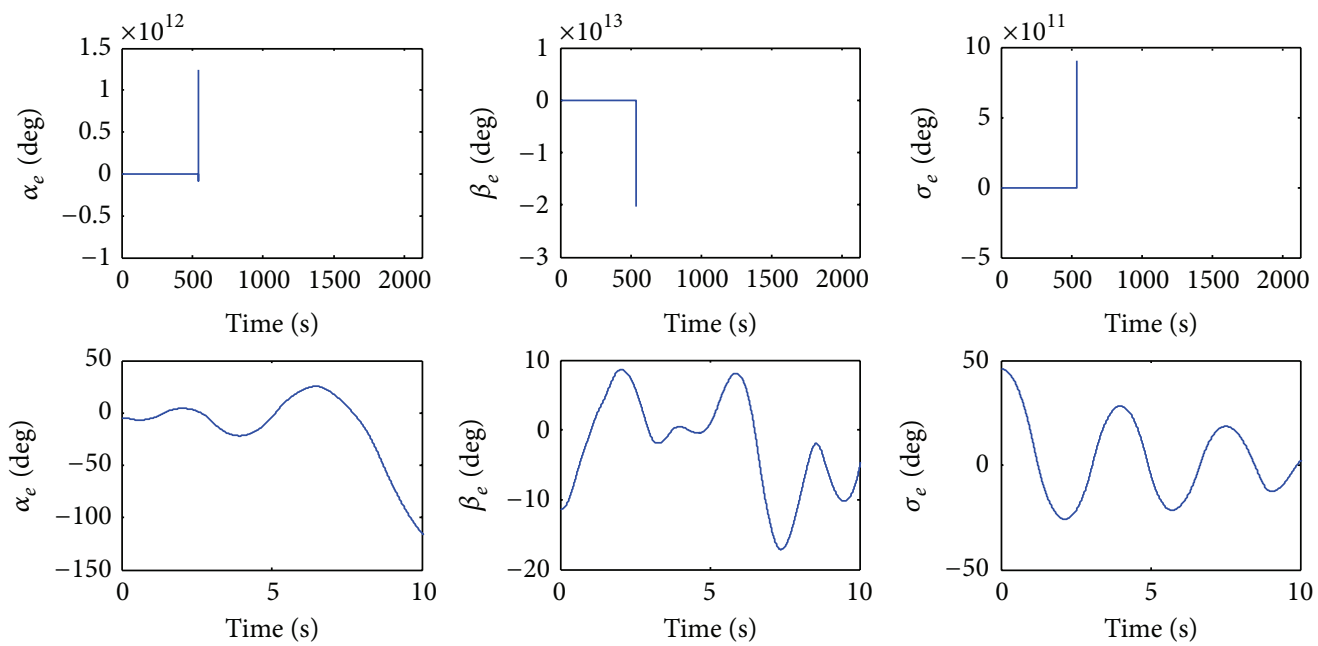

(b) Attitude angle tracking error
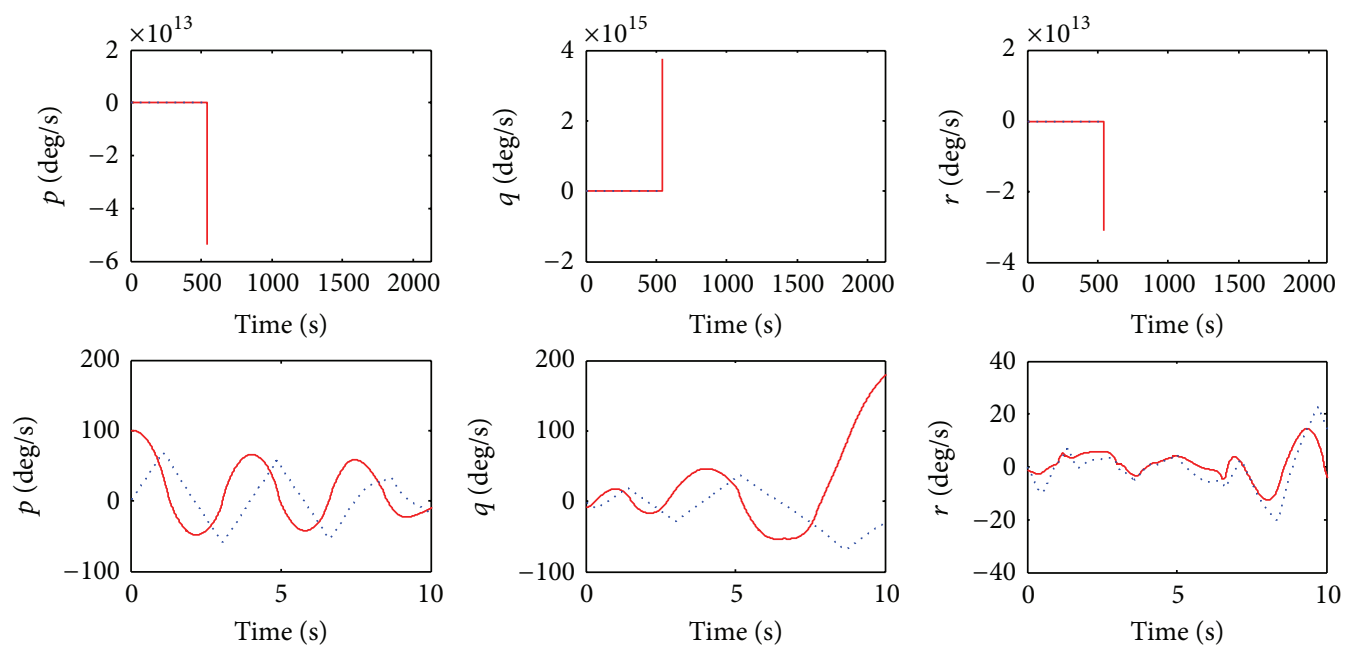

$-p_{d}$
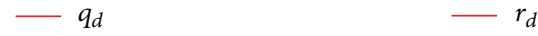

...... Actual value

(c) Attitude angular rate tracking

Figure 3: Continued. 

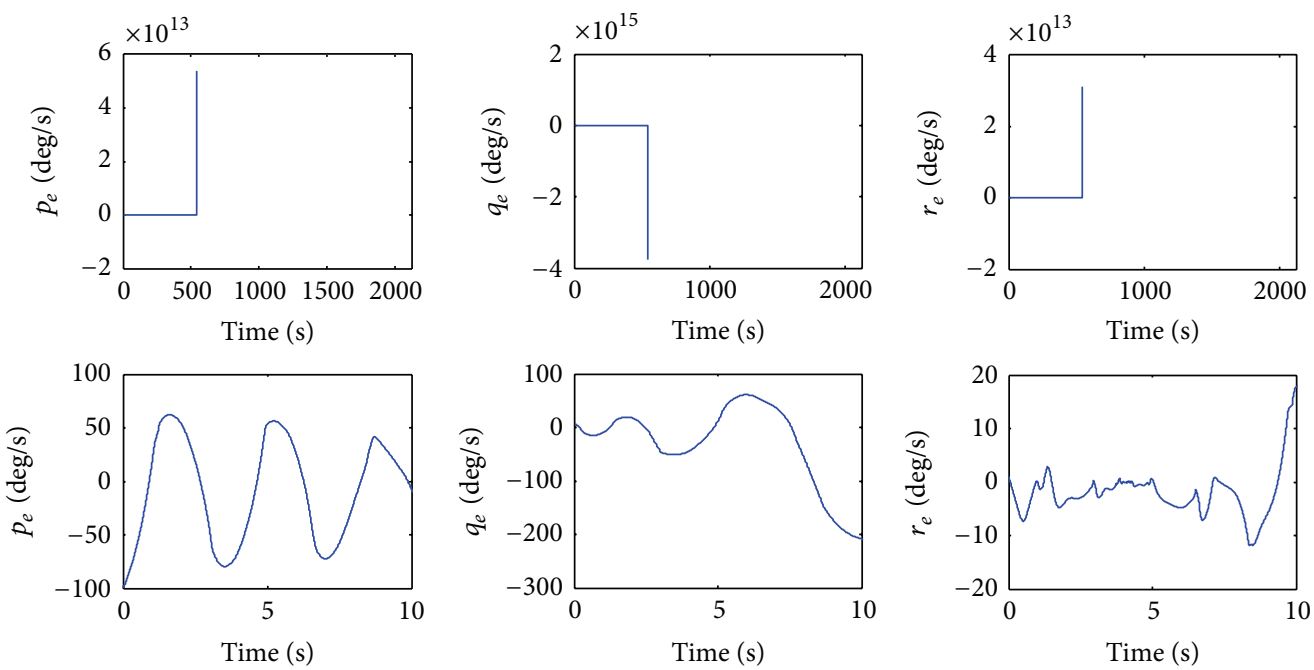

(d) Attitude angular rate tracking error
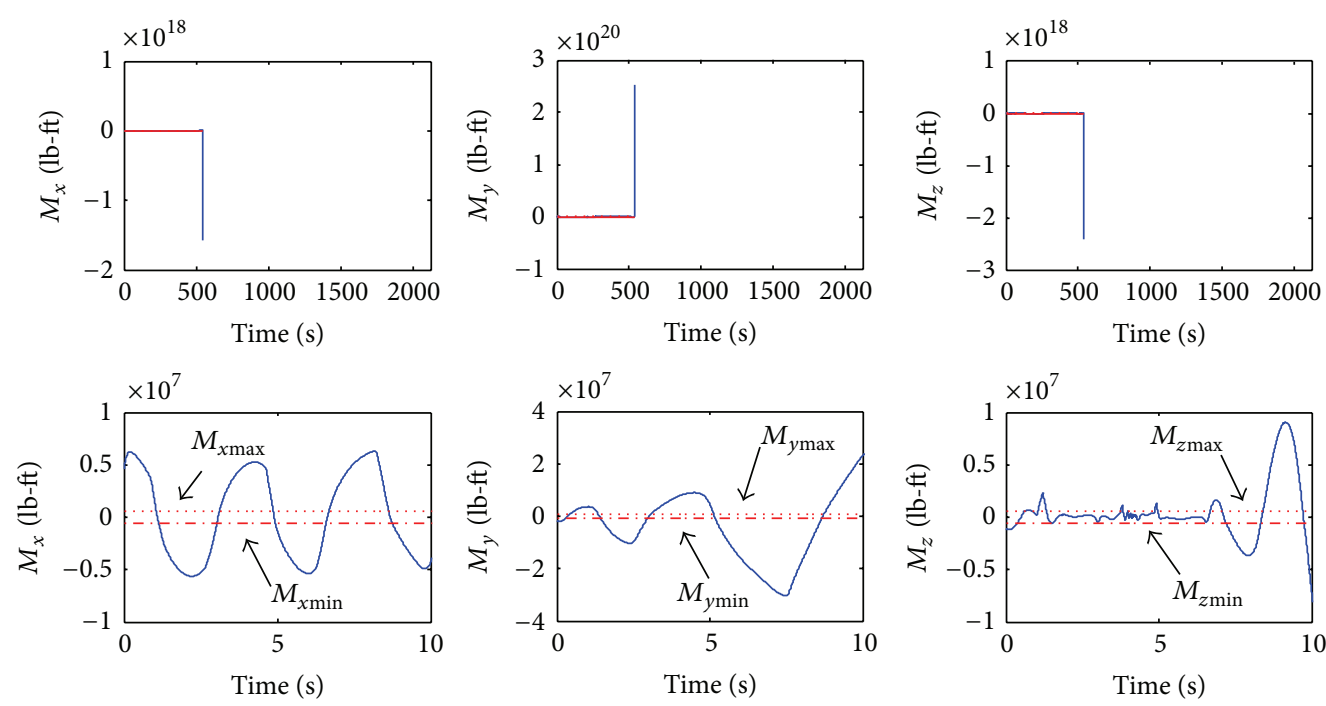

(e) Control inputs

FIGURE 3: Time history of ABFTSMC scheme with manually handling input constraint.

$\mathbf{R z}_{2}+\Delta \mathbf{f}_{1},\left\|\mathbf{R} \mathbf{z}_{2}+\Delta \mathbf{f}_{1}\right\| \leq \sigma_{1}$, the virtual control input $\boldsymbol{\omega}_{d}$ can be designed as

$$
\boldsymbol{\omega}_{d}=\mathbf{R}^{-1}\left(\dot{\mathbf{\Omega}}_{d}-\eta_{1} \operatorname{sgn}\left(\mathbf{s}_{\Omega}\right)\right) .
$$

A sliding mode manifold for attitude angular rate subsystem is chosen as $\mathbf{s}_{\boldsymbol{\omega}}=\mathbf{z}_{2}$, and its time derivative is $\dot{\mathbf{s}}_{\boldsymbol{\omega}}=$ $-\mathbf{I}^{-1} \boldsymbol{\Phi} \mathbf{I} \boldsymbol{\omega}+\mathbf{I}^{-1} \mathbf{u}+\Delta \mathbf{D}_{2}-\dot{\boldsymbol{\omega}}_{d}$. Based on assumption of the uncertain term $\Delta \mathbf{D}_{2},\left\|\Delta \mathbf{D}_{2}\right\| \leq \sigma_{2}$, and the second order filter (26), the control input $\mathbf{u}$ can be designed as

$$
\mathbf{u}=\mathbf{I}\left(\mathbf{I}^{-1} \boldsymbol{\Phi} \mathbf{I} \boldsymbol{\omega}+\overline{\boldsymbol{\omega}}_{d}-\eta_{2} \operatorname{sgn}\left(\mathbf{s}_{\boldsymbol{\omega}}\right)\right),
$$

where $\eta_{1}$ and $\eta_{2}$ are the switching gains, and they satisfy $\eta_{1}>$ $\sigma_{1}>0$ and $\eta_{2}>\sigma_{2}>0$. The sign function would lead to serious chattering.

The controller parameters $\eta_{1}$ and $\eta_{2}$ are selected as $\eta_{1}=$ 20 and $\eta_{2}=70$. Other controller parameters are selected as the same values given in Table 2 . The simulation results applying BOSMC scheme are presented in Figure 2. It shows that the BOSMC scheme can achieve the tracking of guidance command, but there is obvious chattering in attitude angular rate and control inputs. Comparing Figure 2 with Figure 1, it is clear that the undesired chattering of BOSMC resulting from the sign function is effectively reduced by the designed ABFTSMC scheme, and the continuous attitude angular rate and control inputs are obtained.

The attitude tracking performance applying ABFTSMC control strategy manually coping with input constraint is shown in Figure 3. It is obvious that the ABFTSMC scheme cannot achieve stable tracking of attitude angle.

Case 2 (simulation results of CABFTSMC with input constraint, model uncertainty, and external disturbance). In this case, the attitude tracking control problem is simulated to 

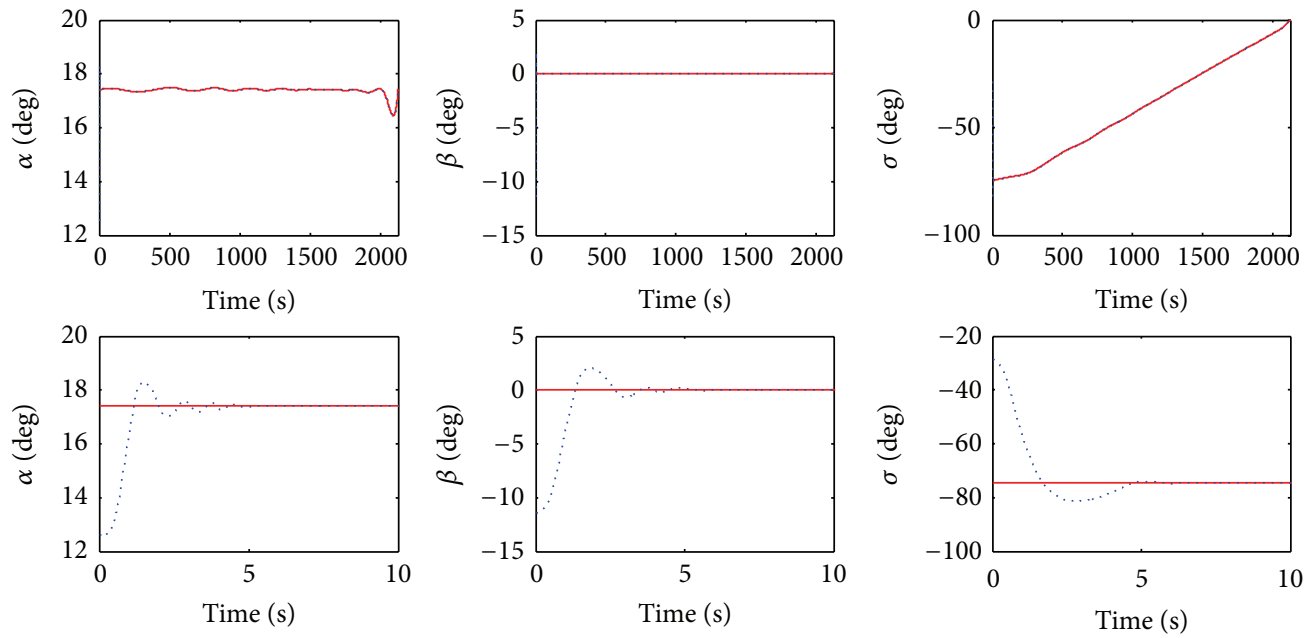

uidance command Actual value

__ Guidance command Actual value

- Guidance command .... Actual value

(a) Attitude angle tracking
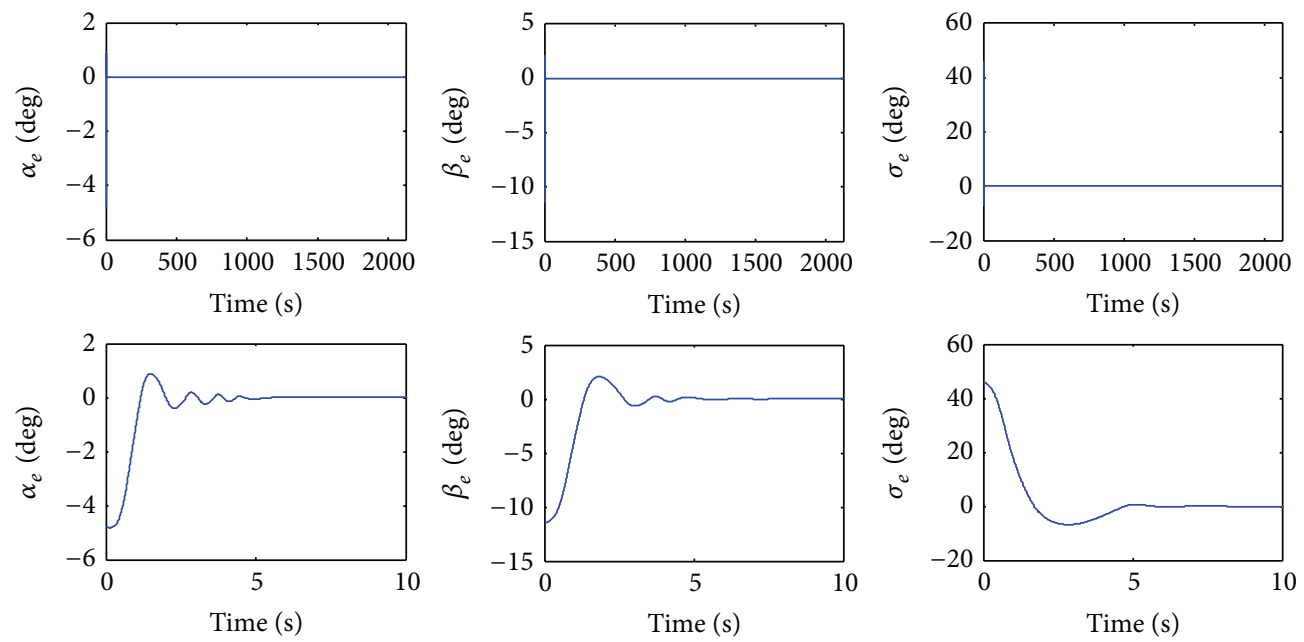

(b) Attitude angle tracking error
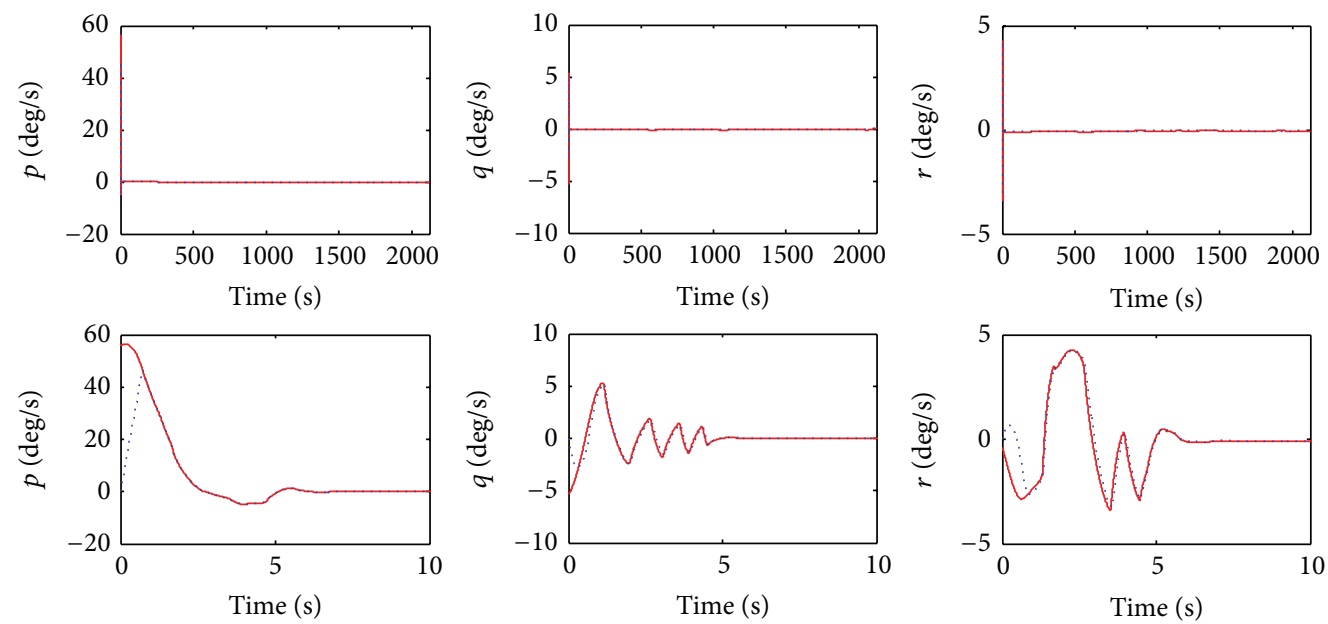

$-p_{d}$

$-q_{d}$

Actual value

$r_{d}$

Actual value

(c) Attitude angular rate tracking

FIgUre 4: Continued. 

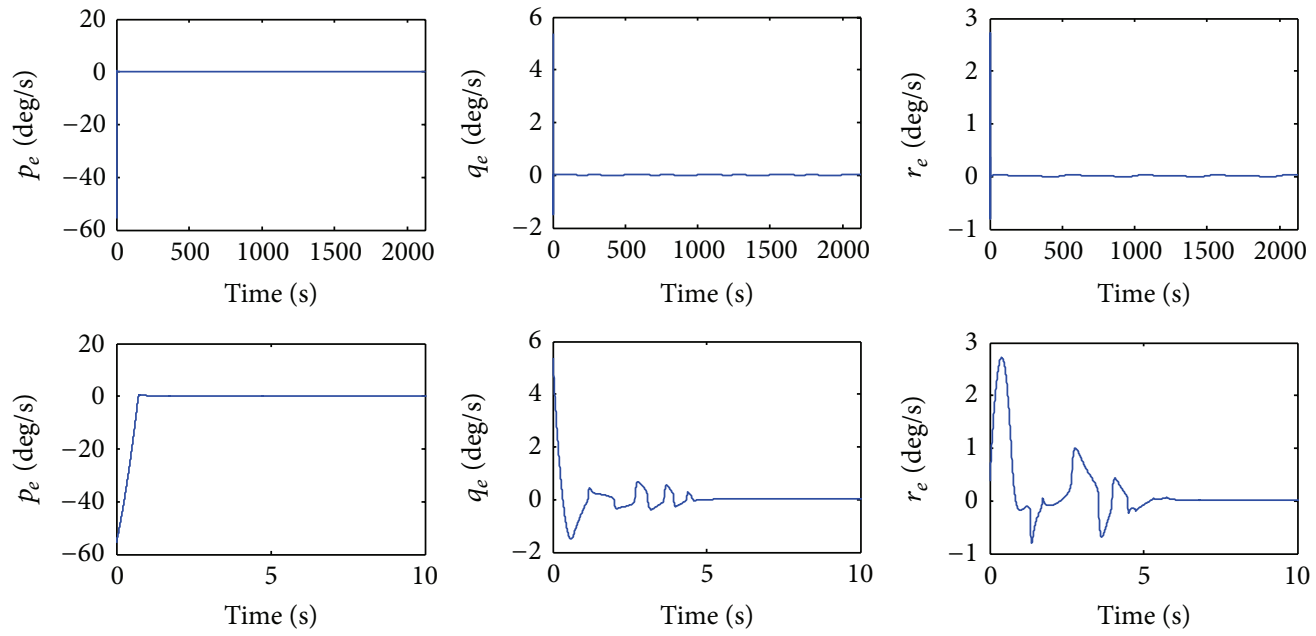

(d) Attitude angular rate tracking error
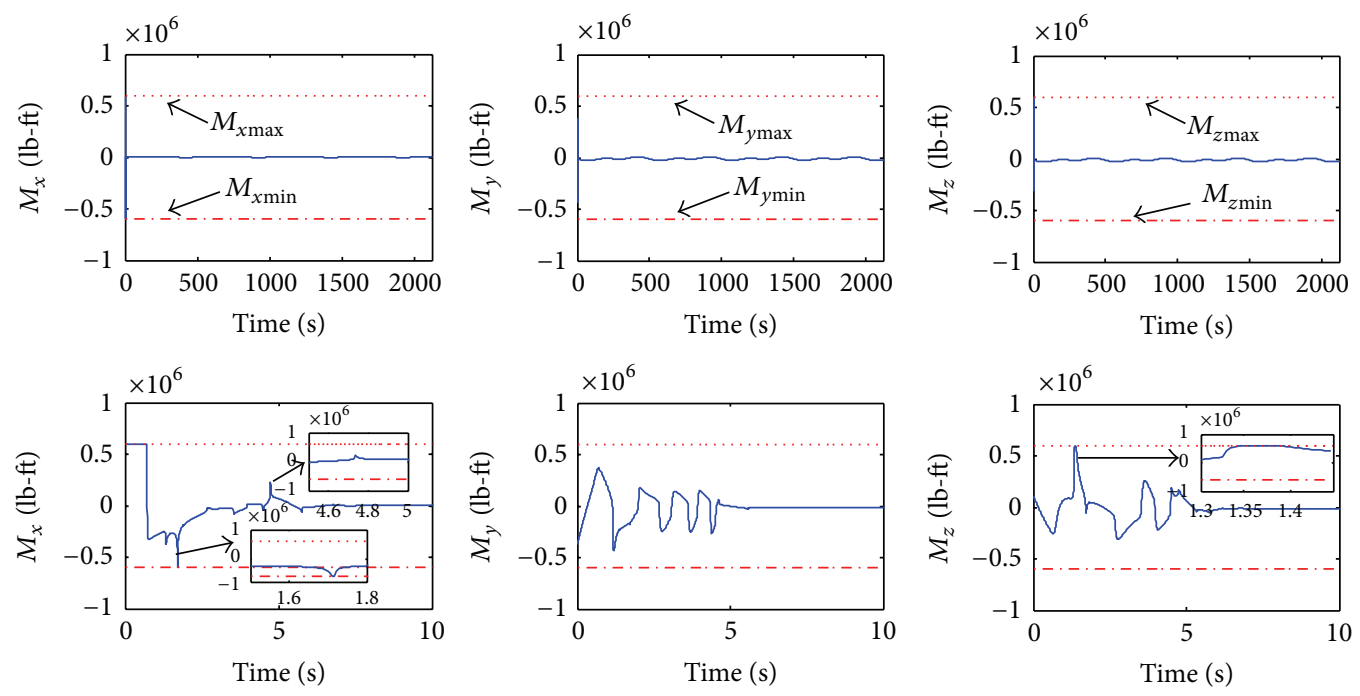

(e) Control inputs

Figure 4: Time history of CABFTSMC scheme.

show the effectiveness of controller (46) in handling the input constraint (40), model uncertainty, and external disturbance. The parameters adopted for the proposed CABFTSMC scheme are given in Table 3. Time histories of attitude angle, attitude angular rate, and control inputs are given in Figure 4. The local time histories are also presented in Figure 4 for better demonstration of dynamic process. It is obvious that the developed CABFTSMC strategy achieves the good tracking performance.

Specifically, from Figures 4(a) and 4(b), the control scheme obtains the desired tracking performance with no obvious steady state error and the tracking error vanishes asymptotically during the whole reentry flight. As demonstrated in Figures 4(c) and 4(d), roll rate, pitch rate, and yaw rate track their reference commands. The tracking errors vanish asymptotically. The behaviors of three control inputs are smooth as can be seen in Figure 4(e). Saturations occur in three control inputs, but they only occur in transient state process. Note that the saturation time is smaller than the transient time, and the control inputs are all within their limits after this period of time; that is, input saturation no longer occurs.

Comparing the results of ABFTSMC with that of CABFTSMC, we can conclude the following. The CABFTSMC scheme leads to a slower response for attitude angle converging to guidance command than that of the response without input constraint under ABFTSMC scheme from Figures 1(a) and 4(a). Despite having a longer response, the proposed CABFTSMC scheme can still assure the attitude stabilization and handle the input constraints efficiently according to Figure 4(e).

The above results suggest that it is necessary and worthwhile to consider input constraint (40) during control design process. Besides, it verifies the effectiveness of the proposed 
TABLE 1: Initial flight condition of RLV.

\begin{tabular}{lc}
\hline State & Value \\
\hline Altitude $h_{0}(\mathrm{ft})$ & 260000 \\
Velocity $v_{0}(\mathrm{ft} / \mathrm{s})$ & 24061 \\
Longitude $\phi_{0}(\mathrm{deg})$ & 0 \\
Latitude $\theta_{0}(\mathrm{deg})$ & 0 \\
FPA $\gamma_{0}(\mathrm{deg})$ & -1.064 \\
Heading angle $\chi_{0}(\mathrm{deg})$ & 0 \\
AOA $\alpha_{0}(\mathrm{deg})$ & 17.41 \\
Sideslip angle $\beta_{0}(\mathrm{deg})$ & 0 \\
Bank angle $\sigma_{0}(\mathrm{deg})$ & -74.48 \\
Roll rate $p(\mathrm{deg} / \mathrm{s})$ & 0 \\
Pitch rate $q(\mathrm{deg} / \mathrm{s})$ & 0 \\
Yaw rate $r(\mathrm{deg} / \mathrm{s})$ & 0 \\
\hline
\end{tabular}

TABLE 2: Controller parameters of ABFTSMC.

\begin{tabular}{lc}
\hline Parameter & Value \\
\hline$m_{1}, m_{2}$ & 5 \\
$n_{1}, n_{2}$ & 7 \\
$\mu_{1}, \mu_{2}$ & 0.1 \\
$\psi_{1}, \psi_{2}$ & 0.2 \\
$\lambda_{1}, \lambda_{2}$ & 1 \\
$\lambda_{3}, \lambda_{4}$ & 4 \\
$\tau_{1}, \tau_{2}$ & 0.2 \\
$\sigma_{1}, \sigma_{2}$ & 0.01 \\
$\varepsilon_{1}$ & 6 \\
$\varepsilon_{2}$ & 10 \\
$\varepsilon_{0 i}, \varepsilon_{1 i}$ & 0.1 \\
$\varepsilon_{\hat{\rho}_{1}}, \varepsilon_{\hat{\rho}_{2}}$ & 0.1 \\
\hline
\end{tabular}

TABLE 3: Controller parameters of CABFTSMC.

\begin{tabular}{lc}
\hline Parameter & Value \\
\hline$m_{1}$ & 5 \\
$n_{1}$ & 7 \\
$\mu_{1}, \mu_{3}, \mu_{4}$ & 0.1 \\
$\varepsilon_{1}$ & 6 \\
$\lambda_{1}$ & $0.3 \operatorname{diag}(0.5,1,1)$ \\
$\lambda_{2}$ & 0.8 \\
$\lambda_{5}$ & 1 \\
$\varepsilon_{\hat{\rho}_{1}}, \varepsilon_{\hat{\rho}_{2}}$ & 0.1 \\
$\varepsilon_{3}$ & 10 \\
$\varepsilon_{\mathbf{u} 0}$ & 0.05 \\
$k_{01}, k_{02}, k_{11}, k_{12}$ & 0.001 \\
$\varepsilon_{0 i}, \varepsilon_{1 i}$ & 0.1 \\
\hline
\end{tabular}

CABFTSMC scheme in providing stable tracking of the guidance command and handling the input constraint.

\section{Conclusion}

In the paper, the finite time attitude controller based on adaptive backstepping control and fast terminal sliding mode control is designed for RLV in reentry phase in spite of input constraint, model uncertainty, and external disturbance. The developed ABFTSMC scheme assures the stable tracking of the attitude angle with model uncertainty and external disturbance. Then, CABFTSMC strategy further considers input constraint. The bounds of uncertain terms are estimated by the adaptive laws. The "explosion of terms" problem is avoided by using the second order filter. The stability of the closed-loop system is proven within the framework of Lyapunov theory, and the finite time convergence of the tracking error is assured. Simulation results demonstrate that the presented controller has reliability of control for RLV during the reentry phase. In addition, the CABFTSMC scheme does not only cope with model uncertainty and external disturbance but also deal with the input constraint effectively. Future research plans include the transformation of control torque to control rudders and controller design for a flexible hypersonic vehicle with input constraint.

\section{Conflict of Interests}

The authors declare that there is no conflict of interests regarding the publication of this paper.

\section{Acknowledgments}

The authors would like to greatly appreciate the editor and all the anonymous reviewers for their comments, which help to improve the quality of this paper. This work has been supported by Key Grant Project of Chinese Ministry of Education (61203012), National Natural Science Foundation of China (91016018, 311012, and 61273092), Tianjin Research Program of Application Foundation and Advanced Technology (11JCZDJC25100 and 12JCZDJC30300), and Aeronautical Science Foundation of China (20125848004), supported by Science and Technology on Aircraft Control Laboratory.

\section{References}

[1] S. Mathavaraj, O. Halbe, and R. Padhi, "Robust control of a reusable launch vehicle in reentry phase using model following neuro-adaptive design," in Proceedings of the AIAA Guidance, Navigation, and Control Conference, AIAA-2010-8312, pp. 2-5, Toronto, Canada, August 2010.

[2] A. S. Hodel and C. E. Hall, "Variable-structure PID control to prevent integrator windup," IEEE Transactions on Industrial Electronics, vol. 48, no. 2, pp. 442-451, 2001.

[3] R. Smith, A. Ahmed, and F. Hadaegh, "The design of a robust parametrically varying attitude controller for the X-33 vehicle," in Proceedings of the AIAA Guidance, Navigation, and Control Conference and Exhibit, AIAA-2000-4158, Denver, Colo, USA, 2000.

[4] J. Georgie and J. Valasek, "Evaluation of longitudinal desired dynamics for dynamic-inversion controlled generic reentry vehicles," Journal of Guidance, Control, and Dynamics, vol. 26, no. 5, pp. 811-819, 2003. 
[5] D. Ito, D. T. Ward, and J. Valasek, "Robust dynamic inversion controller design and analysis for the X-38," in Proceedings of the AIAA Conference on Guidance, Navigation and Control, AIAA2001-4380, Montreal, Canada, 2001.

[6] E. N. Johnson and A. J. Calise, "Limited authority adaptive flight control for reusable launch vehicles," Journal of Guidance, Control, and Dynamics, vol. 26, no. 6, pp. 906-913, 2003.

[7] J. J. Zhu, A. S. Hodel, K. Funston, and C. E. Hall, "X-33 entry flight control design by trajectory linerization-a singular perturbation approach," in Proceedings of the American Astronautical Society Guidance and Control Conference, pp. 151-170, Breckenridge, Colo, USA, February 2001.

[8] T. Bevacqua, E. Best, A. Huizenga, D. Cooper, and J. J. Zhu, "Improved trajectory linearization flight controller for reusable launch vehicles," in Proceedings of the 42nd AIAA Aerospace Sciences Meeting and Exhibit, AIAA-2004-875, pp. 11242-11253, Reno, Nev, USA, January 2004.

[9] J. J. Zhu, "Well-defined series and parallel D-spectra for linear time-varying systems," in Proceedings of the American Control Conference, pp. 734-738, Baltimore, Md, USA, July 1994.

[10] Y. B. Shtessel, J. McDuffie, and M. Jackson, "Sliding mode control of the X-33 vehicle in launch and re-entry modes," in Proceedings of the AIAA Guidance, Navigation and Control Conference and Exhibit, AIAA-1998-4414, Reston, Va, USA, August 2008.

[11] J. E. Stott and Y. B. Shtessel, "Launch vehicle attitude control using sliding mode control and observation techniques," Journal of the Franklin Institute, vol. 349, no. 2, pp. 397-412, 2012.

[12] M. Xin and S. N. Balakrishnan, "State dependent Riccati equation based spacecraft attitude control," in Proceedings of the AIAA Aerospace Sciences Meeting and Exhibit, AIAA-2002-1071, Reno, Nev, USA, 2002.

[13] Q. M. Lam, P. Krishnamurthy, and F. Khorrami, "Enhancing flight control system performance using SDRE based controller as an augmentation layer," in Proceedings of the AIAA Guidance, Navigation, and Control Conference and Exhibit, AIAA-20096171, Chicago, 1ll, USA, August 2009.

[14] N. O. Pérez-Arancibia, T.-C. Tsao, and J. S. Gibson, "Saturationinduced instability and its avoidance in adaptive control of hard disk drives," IEEE Transactions on Control Systems Technology, vol. 18, no. 2, pp. 368-382, 2010.

[15] V. Kapila and K. Grigoriadis, Actuator Saturation Control (Automation and Control Engineering), CRC Press, 2002.

[16] T. Hu and Z. Lin, Control Systems with Actuator Saturation: Analysis and Design, Birkhäuser, Boston, Mass, USA, 2001.

[17] C. Wen, J. Zhou, Z. Liu, and H. Su, "Robust adaptive control of uncertain nonlinear systems in the presence of input saturation and external disturbance," IEEE Transactions on Automatic Control, vol. 56, no. 7, pp. 1672-1678, 2011.

[18] K. Y. Volyanskyy, W. M. Haddad, and J. M. Bailey, "Neuroadaptive output feedback control for nonlinear nonnegative dynamical systems with actuator amplitude and integral constraints," International Journal of Adaptive Control and Signal Processing, vol. 25, no. 6, pp. 483-501, 2011.

[19] J. H. Huang, C. Y. Wen, W. Wang, and Z. P. Jiang, "Adaptive stabilization and tracking control of a nonholonomic mobile robot with input saturation and disturbance," Systemsv \& Control Letters, vol. 62, no. 3, pp. 234-241, 2013.

[20] D. M. Ge, G. H. Sun, and H. R. Karimi, "Robust Anti-Windup control considering multiple design objectives," Mathematical Problems in Engineering, vol. 2012, Article ID 586279, 13 pages, 2012.
[21] L. Zaccarian and A. R. Teel, Modern Anti-Windup Synthesis: Control Augmentation for Actuator Saturation, vol. 22, Princeton University Press, 2011.

[22] K. P. Groves, A. Serrani, S. Yurkovich, M. A. Bolender, and D. B. Doman, "Anti-windup control for an air-breathing hypersonic vehicle model," in Proceedings of the AIAA Guidance, Navigation, and Control Conference, pp. 3649-3662, AIAA, Keystone, Colo, USA, August 2006.

[23] A. Zinnecker, A. Serrani, M. A. Bolender, and D. B. Doman, "Combined reference governor and anti-windup design for constrained hypersonic vehicles models," in Proceedings of the AIAA Guidance, Navigation, and Control Conference and Exhibit, AIAA-2009-6283, Chicago, III, USA, August 2009.

[24] Y. Lv, Q. Hu, G. Ma, and J. Zhou, "6 DOF synchronized control for spacecraft formation flying with input constraint and parameter uncertainties," ISA Transactions, vol. 50, no. 4, pp. 573-580, 2011.

[25] J. J. Recasens, Q. P. Chu, and J. A. Mulder, "Robust model predictive control of a feedback linearized system for a lifting-body reentry vehicle," in Proceedings of the AIAA Guidance, Navigation, and Control Conference, pp. 2952-2984, San Francisco, Calif, USA, August 2005.

[26] W. R. Van Soest, Q. P. Chu, and J. A. Mulder, "Combined feedback linearization and constrained model predictive control for entry flight," Journal of Guidance, Control, and Dynamics, vol. 29, no. 2, pp. 427-434, 2006.

[27] S. S. Vaddi and P. Sengupta, "Controller design for hypersonic vehicles accommodating nonlinear state and control constraints," in Proceedings of the AIAA Guidance, Navigation, and Control Conference and Exhibit, AIAA-2009-6286, Chicago, III, USA, August 2009.

[28] Y. Gao, "Linear feedback guidance for low-thrust manyrevolution earth-orbit transfers," Journal of Spacecraft and Rockets, vol. 46, no. 6, pp. 1320-1325, 2009.

[29] E. Lavretsky and N. Hovakimyan, "Stable adaptation in the presence of actuator constraints with flight control applications," Journal of Guidance, Control, and Dynamics, vol. 30, no. 2, pp. 337-345, 2007.

[30] Q. M. Lam, D. T. Drake, and D. B. Ridgely, "Input saturation treatments: a performance comparison of direct adaptive control and $\theta$-D control methodologies," in Proceedings of the IEEE Aerospace Conference, pp. 1-15, Big Sky, Mont, USA, March 2007.

[31] L. Sonneveldt, Q. P. Chu, and J. A. Mulder, "Nonlinear flight control design using constrained adaptive backstepping," Journal of Guidance, Control, and Dynamics, vol. 30, no. 2, pp. 322336, 2007.

[32] I. Ali, G. Radice, and J. Kim, "Backstepping control design with actuator torque bound for spacecraft attitude maneuver," Journal of Guidance, Control, and Dynamics, vol. 33, no. 1, pp. 254-259, 2010.

[33] J. Farrell, M. Polycarpou, and M. Sharma, "Adaptive backstepping with magnitude, rate, and bandwidth constraints: aircraft longitude control," in 2003 American Control Conference, pp. 3898-3904, June 2003.

[34] M. Kristic, I. Kanellakopoulos, and P. Kosotovic, Nonlinear and Adaptive Control Design, John Wiley \& Sons, New York, NY, USA, 1995.

[35] P. Kokotović and M. Arcak, "Constructive nonlinear control: a historical perspective," Automatica, vol. 37, no. 5, pp. 637-662, 2001. 
[36] A. Stotsky, J. K. Hedrick, and P. P. Yip, "Use of sliding modes to simplify the backstepping control method," in Proceedings of the American Control Conference, pp. 1703-1708, June 1997.

[37] H. H. Choi, "LMI-based sliding surface design for integral sliding mode control of mismatched uncertain systems," IEEE Transactions on Automatic Control, vol. 52, no. 4, pp. 736-742, 2007.

[38] F. G. Ding, J. Wu, and Y. H. Wang, "Stabilization of an underactuated surface vessel based on adaptive sliding mode and backstepping control," Mathematical Problems in Engineering, vol. 2013, Article ID 324954, 5 pages, 2013.

[39] Z. Wang, "Synchronization of an uncertain fractional-order chaotic system via backstepping sliding mode control," Discrete Dynamics in Nature and Society, vol. 2013, Article ID 732503, 6 pages, 2013.

[40] H. Shi, Y. Feng, and X. Yu, "Adaptive backstepping hybrid terminal sliding-mode control for permanent magnet synchronous motor," in Proceedings of the 11th International Workshop on Variable Structure Systems (VSS '10), pp. 272-276, Mexico City, Mexico, June 2010.

[41] C.-C. Peng, A. W.-J. Hsue, and C.-L. Chen, "Variable structure based robust backstepping controller design for nonlinear systems," Nonlinear Dynamics, vol. 63, no. 1-2, pp. 253-262, 2011.

[42] P. N. Desai and B. A. Conway, "Six-degree-of-freedom trajectory optimization using a two-timescale collocation architecture," Journal of Guidance, Control, and Dynamics, vol. 31, no. 5, pp. 1308-1315, 2008.

[43] K. P. Bollino, High-fidelity real-time trajectory optimization for reusable launch vehicles [dissertation], Naval Postgraduate School, Monterey, Calif, USA, 2006.

[44] X. Yu and M. Zhihong, "Fast terminal sliding-mode control design for nonlinear dynamical systems," IEEE Transactions on Circuits and Systems I, vol. 49, no. 2, pp. 261-264, 2002.

[45] K. Y. Zhang, Research on some problems of the theory and application of variable structure control [dissertation], Zhejiang University, Hangzhou, China, 2002.

[46] D. Swaroop, J. C. Gerdes, P. P. Yip, and J. K. Hedrick, "Dynamic surface control of nonlinear systems," in Proceedings of the American Control Conference, pp. 3028-3034, June 1997.

[47] D. Swaroop, J. K. Hedrick, P. P. Yip, and J. C. Gerdes, "Dynamic surface control for a class of nonlinear systems," IEEE Transactions on Automatic Control, vol. 45, no. 10, pp. 1893-1899, 2000.

[48] M. Chen, S. S. Ge, and B. V. E. How, "Robust adaptive neural network control for a class of uncertain MIMO nonlinear systems with input nonlinearities," IEEE Transactions on Neural Networks, vol. 21, no. 5, pp. 796-812, 2010.

[49] M. Chen, S. S. Ge, and B. Ren, "Adaptive tracking control of uncertain MIMO nonlinear systems with input constraints," Automatica, vol. 47, no. 3, pp. 452-465, 2011.

[50] C.-Y. Li, W.-X. Jing, and C.-S. Gao, "Adaptive backsteppingbased flight control system using integral filters," Aerospace Science and Technology, vol. 13, no. 2-3, pp. 105-113, 2009.

[51] J. D. Bošković, S.-M. Li, and R. K. Mehra, "Robust adaptive variable structure control of spacecraft under control input saturation," Journal of Guidance, Control, and Dynamics, vol. 24, no. 1, pp. 14-22, 2001.

[52] Q. Hu, "Robust adaptive sliding mode attitude maneuvering and vibration damping of three-axis-stabilized flexible spacecraft with actuator saturation limits," Nonlinear Dynamics, vol. 55, no. 4, pp. 301-321, 2009.
[53] G. Wheeler, C.-Y. Su, and Y. Stepanenko, "A sliding mode controller with improved adaptation laws for the upper bounds on the norm of uncertainties," Automatica, vol. 34, no. 12, pp. 1657-1661, 1998.

[54] A. Levant, "Higher order sliding modes and arbitrary-order exact robust differentiation," in Proceedings of the European Control Conference, pp. 996-1001, Porto, Portugal, September 2001.

[55] B. Tian and Q. Zong, "Optimal guidance for reentry vehicles based on indirect Legendre pseudospectral method," Acta Astronautica, vol. 68, no. 7-8, pp. 1176-1184, 2011. 


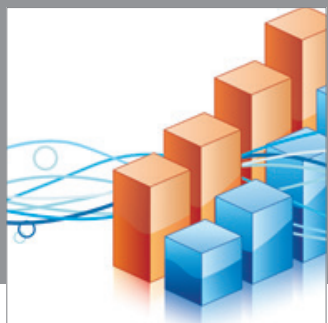

Advances in

Operations Research

mansans

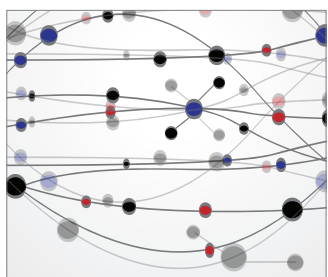

The Scientific World Journal
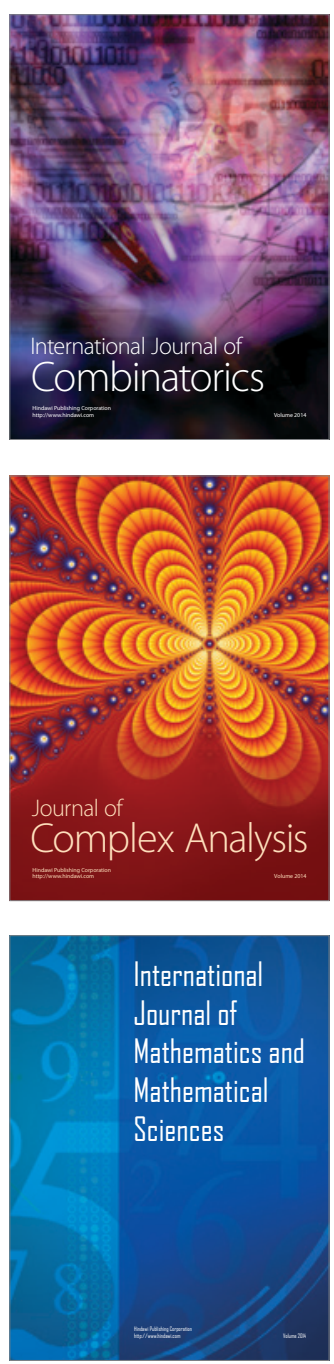
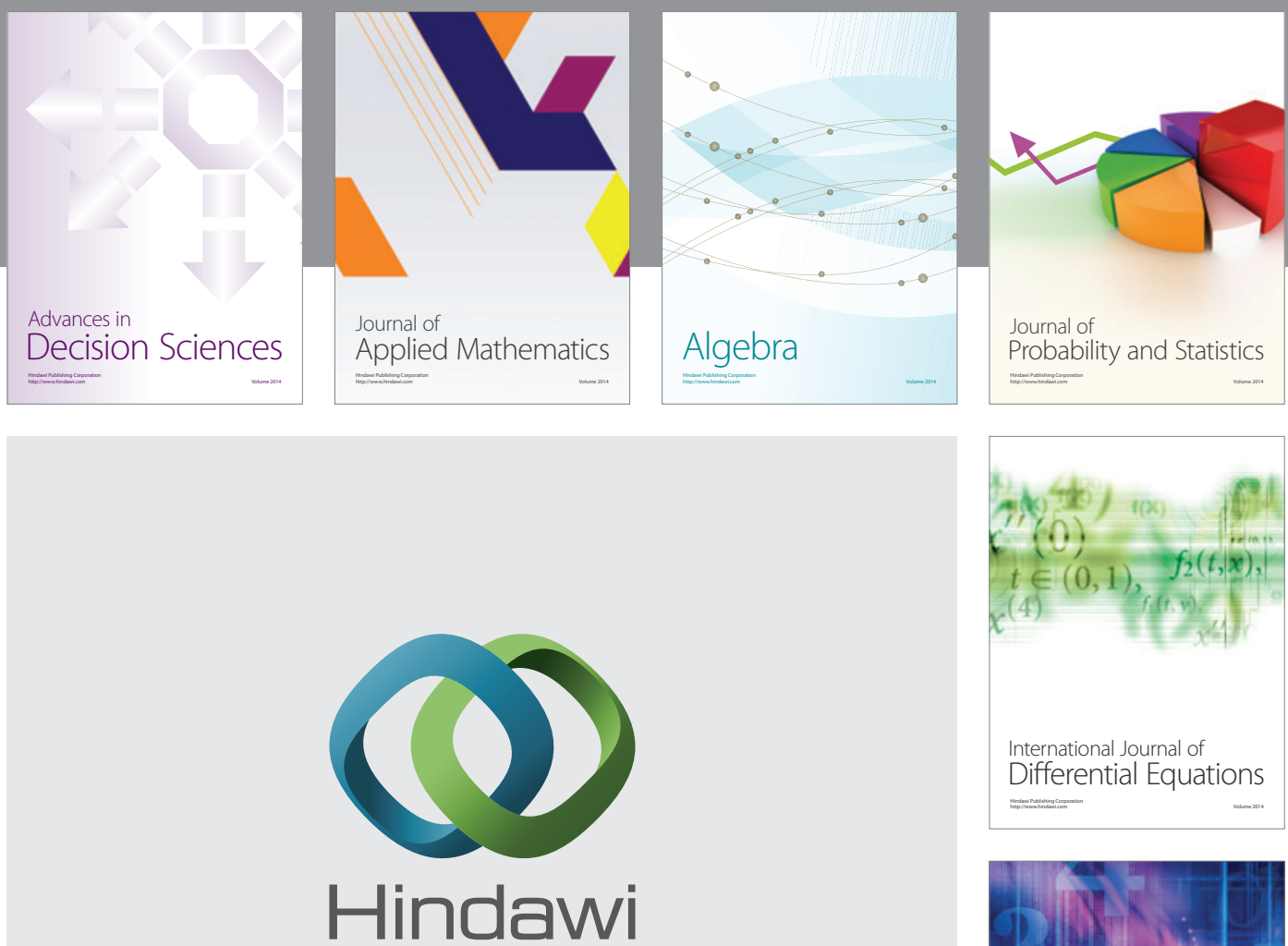

Submit your manuscripts at http://www.hindawi.com
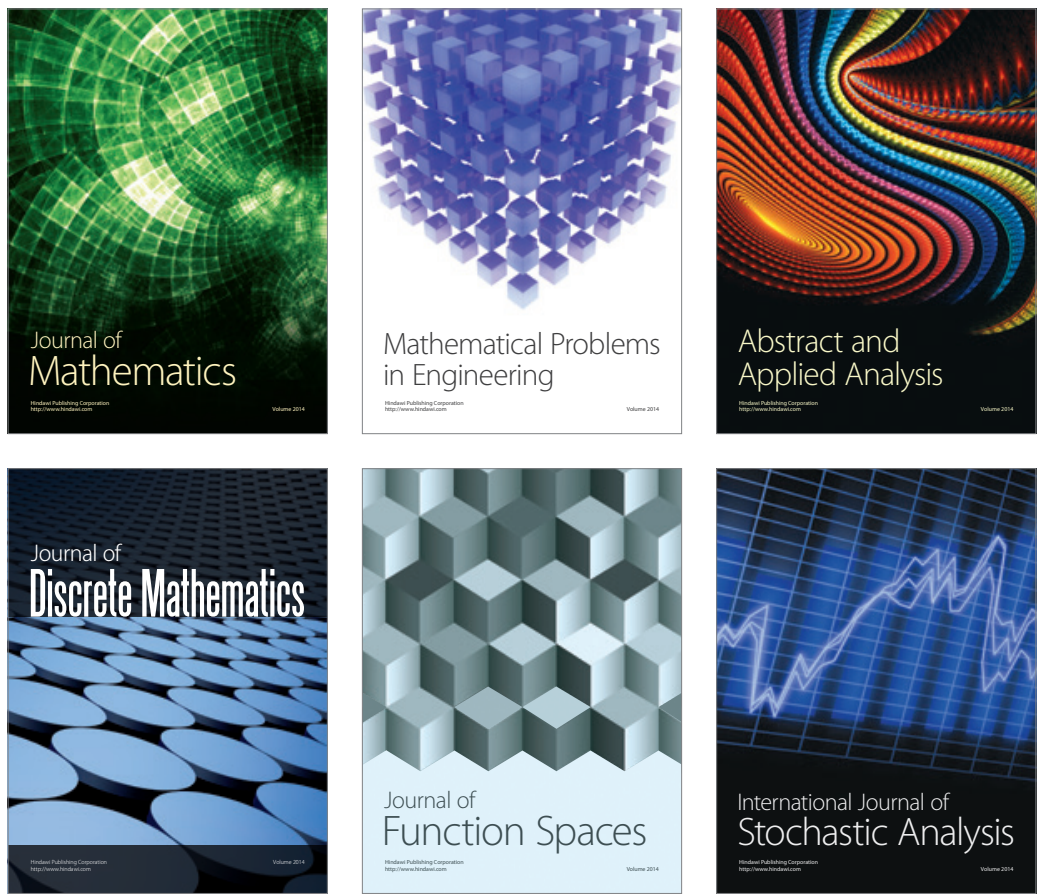

Journal of

Function Spaces

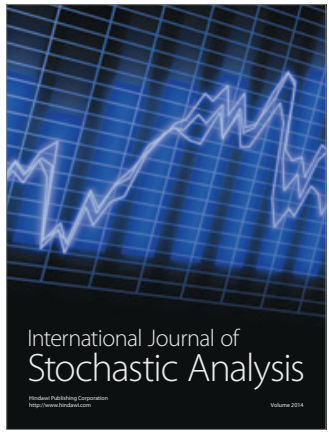

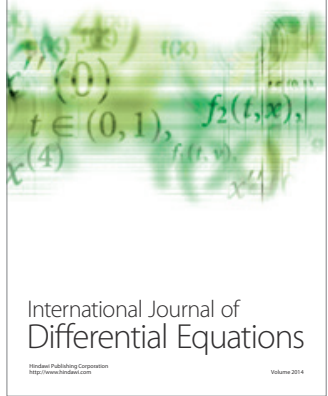
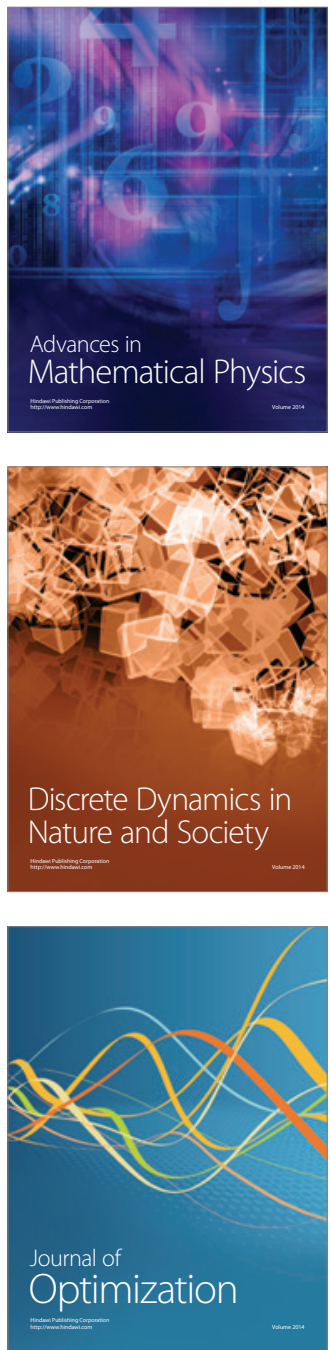